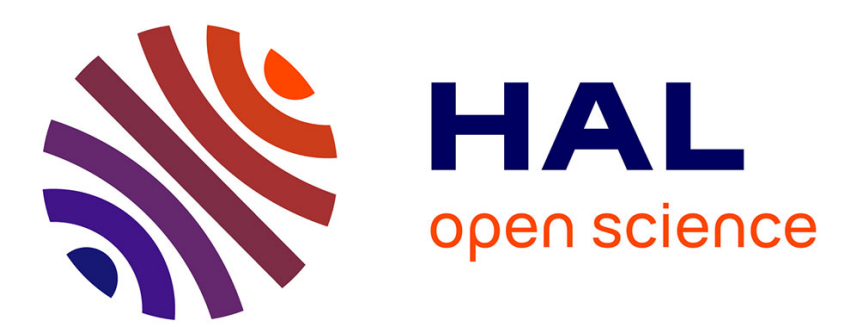

\title{
Micro-Roughness Effects in (Elasto)Hydrodynamic Lubrication Including a Mass-Flow Preserving Cavitation Model
}

\author{
Guy Bayada, Sébastien Martin, Carlos Vazquez
}

\section{To cite this version:}

Guy Bayada, Sébastien Martin, Carlos Vazquez. Micro-Roughness Effects in (Elasto)Hydrodynamic Lubrication Including a Mass-Flow Preserving Cavitation Model. 2005. hal-00008355v1

\section{HAL Id: hal-00008355 \\ https://hal.science/hal-00008355v1}

Preprint submitted on 2 Sep 2005 (v1), last revised 22 Feb 2007 (v2)

HAL is a multi-disciplinary open access archive for the deposit and dissemination of scientific research documents, whether they are published or not. The documents may come from teaching and research institutions in France or abroad, or from public or private research centers.
L'archive ouverte pluridisciplinaire HAL, est destinée au dépôt et à la diffusion de documents scientifiques de niveau recherche, publiés ou non, émanant des établissements d'enseignement et de recherche français ou étrangers, des laboratoires publics ou privés. 


\title{
Micro-Roughness Effects in (Elasto)Hydrodynamic Lubrication Including a Mass-Flow Preserving Cavitation Model
}

\author{
Guy Bayada \\ INSA de Lyon / LAMCOS CNRS-UMR 5514 / ICJ CNRS-UMR 5208 \\ Bât. Léonard de Vinci, 21 av. Jean Capelle \\ 69621 Villeurbanne cedex, France
}

Sébastien Martin

INSA de Lyon / ICJ CNRS-UMR 5208

Bât. Léonard de Vinci, 21 av. Jean Capelle

69621 Villeurbanne cedex, France

Carlos Vázquez

Universidade da Coruña

Facultade de Informática / Dept. de Matemáticas

Campus Elviña, 15071-A Coruña, España

\begin{abstract}
An average Reynolds equation is proposed for predicting the effects of deterministic periodic roughness, taking JFO mass flow preserving cavitation model and elastohydrodynamic effects into account. For this, the asymptotic model is based upon double scale analysis approach. The average Reynolds equation can be used both for microscopic interasperity cavitation and macroscopic one. The validity of such a model is verified by numerical experiments.
\end{abstract}

Key words: Elastohydrodynamic; Lubrication; Roughness.

Email addresses: guy.bayada@insa-lyon.fr (Guy Bayada), sebastien.martin@insa-lyon.fr (Sébastien Martin), carlosv@udc.es (Carlos Vázquez). 


\section{NOMENCLATURE}

$$
\begin{aligned}
& h_{r} \quad=\text { rigid gap } \\
& h_{[p]} \quad=\text { effective gap (including deformation) } \\
& h_{0} \quad=\text { minimum thickness of the rigid gap } \\
& R=\text { sphere or cylinder section radius } \\
& k=\text { Hertz kernel } \\
& p \quad=\text { pressure } \\
& p_{0}, p_{1} \ldots=\text { approximations of the pressure } \\
& \theta \quad=\text { saturation } \\
& \theta_{0} \quad=\text { microscopic homogenized saturation } \\
& \Theta_{i}, \Theta \quad=\text { macro-homogenized saturations } \\
& x=\left(x_{1}, x_{2}\right) \quad=\text { space variables } \\
& y=\left(y_{1}, y_{2}\right) \quad=\text { microscale variables } \\
& Y=] 0,1[\times] 0,1[\quad=\text { rescaled microcell } \\
& \left(A_{[p]}^{\star}\right)_{i j}, A_{[p]}^{(i, \star)}=\text { homogenized coefficients } \\
& B_{[p]}^{(i, \star)}, B_{[p]}^{(i, 0)}=\text { homogenized coefficients } \\
& w_{\left[p_{0}\right]}^{(i)}, \chi_{\left[p_{0}\right]}^{(i, 0)} \quad=\text { auxiliary functions defined on } Y \\
& \alpha \quad=\text { piezoviscosity coefficient } \\
& \partial / \partial n=\text { normal derivative } \\
& \varepsilon \quad=\text { roughness spacing } \\
& \leftarrow^{Y} \quad=\text { average operator with respect to } y \\
& L \quad=\text { length of the journal bearing } \\
& R_{b} \quad=\text { bearing radius } \\
& R_{j} \quad=\text { journal radius } \\
& R_{m} \quad=\text { mean radius } \\
& \Gamma_{a}, \Gamma_{0}, \Gamma_{\sharp}, \Gamma_{\star}=\text { boundaries of the device } \\
& c \quad=\text { clearance } \\
& \rho \quad=\text { eccentricity of the bearing } \\
& p_{a} \quad=\text { supply pressure } \\
& \mu \quad=\text { viscosity } \\
& v_{0} \quad=\text { velocity of the hearing } \\
& a_{r} \quad=\text { amplitude of the roughness } \\
& W \quad=\text { load } \\
& \theta_{\text {in }} \quad=\text { supply flow }
\end{aligned}
$$




\section{Introduction}

In this paper, it is explained how the double scale procedure, already used to obtain average equations with periodic roughness in the case of rigid bearings [1-3], can be extended to EHD problems including cavitation and starvation. The JFO mass flow preserving model is used, including pressure and saturation as unknown functions. This model takes into account both microcavitation (due to the microroughness) and macrocavitation (due to the diverging part of the gap). Average equation can be easily solved for some specific roughness patterns (transverse, longitudinal) exactly in the same way as the initial EHD problem with cavitation. Numerical results are given for both purely hydrodynamic and EHD point-contact problems, for a two dimensional device.

\section{Basic equations}

Our studied cavitation model, like the Elrod algorithm and its variants [4,5], views the film as a mixture. It does not, however, make the assumption of liquid compressibility in the full film area as in [6] and some other papers. The flow obeys the following "universal" Reynolds equation (here written in a dimensionless form) through all the gap in which the pressure cavitation is assumed to be zero in the cavitation area. Moreover, the lubricant is piezoviscous so that the viscosity obeys the Barus law (notice that other laws may be taken into account). The effective gap contains a rigid contribution and an elastic one, which is given by the Hertz law (for local contacts):

$$
\begin{gathered}
\sum_{i=1}^{2} \frac{\partial}{\partial x_{i}}\left(h_{[p]}^{3} e^{-\alpha p} \frac{\partial p}{\partial x_{i}}\right)=\frac{\partial \theta h_{[p]}}{\partial x_{1}} \\
p \geq 0 \\
0 \leq \theta \leq 1 \\
p(1-\theta)=0
\end{gathered}
$$

$p$ is the pressure (assumed to be a positive function), $\theta$ is the relative mixture density, $h_{[p]}$ the real film thickness, $x_{1}$ is the direction of the effective relative shear velocity of the device, while $x_{2}$ is the transverse direction. Here, $h_{[p]}$, which is the effective gap between two close surfaces, contains a given rigid contribution $h_{r}$ and an elastic one, which strongly depends on the main unknown $p$ (lubricant pressure) in the following nonlocal form:

$$
h_{[p]}(x)=h_{r}(x)+\int_{\Omega} k(x, z) p(z) d z,
$$


the kernel $k$ depending on the kind of contact. The classical approximation of the rigid gap [7] is given by the expression

$$
h_{r}(x)= \begin{cases}h_{0}+\frac{x_{1}^{2}+x_{2}^{2}}{2 R}, & \text { for ball bearings }, \\ h_{0}+\frac{x_{1}^{2}}{R}, & \text { for linear bearings }\end{cases}
$$

that represents a parabolic approximation for a given sphere-plane (point contact) or cylinder-plane (line contact) gap, $R$ being the sphere or cylinder section radius. The positive constant $h_{0}$ corresponds to the gap at the point nearest to contact. Now, let us introduce the general property of the kernel $k$ :

$$
k(x, z)= \begin{cases}c_{0} \log \left|\frac{c_{1}-z_{1}}{x_{1}-z_{1}}\right|, & \text { for line contacts } \\ \frac{c_{0}}{\sqrt{\left(x_{1}-z_{1}\right)^{2}+\left(x_{2}-z_{2}\right)^{2}}}, & \text { for point contacts }\end{cases}
$$

where $c_{0}>0$ and $c_{1} \geq \max \left\{\left|x_{1}\right|, x \in \bar{\Omega}\right\}$.

\section{Asymptotic expansion}

Let us suppose that the roughness is periodically reproduced in the two $x_{1}$ and $x_{2}$ directions from an elementary cell $Y$ (or "miniature bearing" in Tonder's terminology). We denote by $\varepsilon$ the ratio of the homothetic transformation passing from the elementary cell $Y=Y_{1} \times Y_{2}$ to the real bearing and by $y_{1}=x_{1} / \varepsilon$ and $y_{2}=x_{2} / \varepsilon$ the local variables (see FIG. 1).

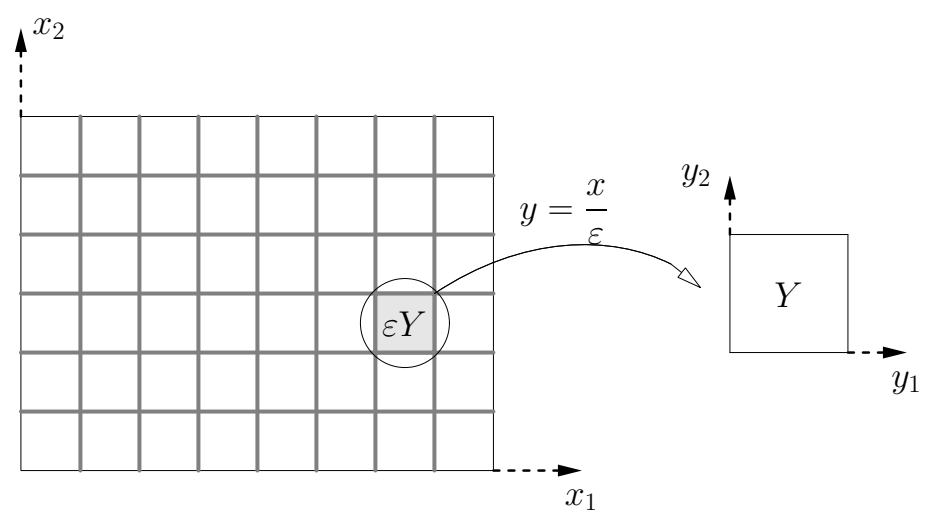

Fig. 1. Macroscopic domain and elementary cells

Let us now consider gaps that can be written as $h_{r}(x, x / \varepsilon)$. Introducing now the fast variables $y_{1}$ and $y_{2}$, it appears that the new expression for the gap 
is $h_{r}(x, y)$ in the two-scale approach. The combined computation in terms of $\left(x_{1}, x_{2}\right)$ or $\left(y_{1}, y_{2}\right)$ is an important feature of the method. It is convenient to consider first $x$ and $y$ as independent variables and to replace next $y$ by $x / \varepsilon$ (see $[1])$.

In this section, we recall the main tools that allow the derivation of average equations. The process has the same feature as the one corresponding to the hydrodynamic case, which can be found in [3]. The equations should be read as

$$
\begin{gathered}
\sum_{j=1}^{2} \frac{\partial}{\partial x_{j}}\left\{\left(h_{[p]}\left(x, \frac{x}{\varepsilon}\right)\right)^{3} e^{-\alpha p} \frac{\partial p}{\partial x_{j}}\right\}=\frac{\partial}{\partial x_{1}}\left\{\theta h_{[p]}\left(x, \frac{x}{\varepsilon}\right)\right\}, \\
p \geq 0, \quad 0 \leq \theta \leq 1, \quad p(1-\theta)=0
\end{gathered}
$$

with the additional notation:

$$
h_{[p]}\left(x, \frac{x}{\varepsilon}\right)=h_{r}\left(x, \frac{x}{\varepsilon}\right)+\int_{\Omega} k(x, z) p(z) d z .
$$

We shall look for an asymptotic expansion of the solutions:

$$
\begin{aligned}
& p(x)=p_{0}(x)+\varepsilon p_{1}\left(x, \frac{x}{\varepsilon}\right)+\varepsilon^{2} p_{2}\left(x, \frac{x}{\varepsilon}\right)+\ldots, \\
& \theta(x)=\theta_{0}\left(x, \frac{x}{\varepsilon}\right)
\end{aligned}
$$

$p_{0}$ being a positive function, and each unknown $p_{i}(i \geq 1)$ and $\theta_{0}$ being function of $(x, y)$. The problem of the boundary conditions to be satisfied by the $p_{i}$ is somewhat difficult but may be summarized so:

(i) The natural boundary conditions on $(p, \theta)$ are assigned to $p_{0}$ and an equivalent saturation linked to $\theta_{0}$, which will be developped in next subsection.

(ii) The function $p_{i}, i \geq 1$, are $Y$ periodic, i.e. periodic in the two variables $y_{1}$, $y_{2}$, for each value of $\left(x_{1}, x_{2}\right)$.

To be noticed that, unlike of $p$, we do not introduce an asymptotic expansion for $\theta$. This can be explained by observing the evolution of $p$ and $\theta$ as $\varepsilon$ tends to 0 . Clearly, the oscillations of the pressure are decreasing and $p$ tends to a smooth function (namely $p_{0}$ which, actually, does not depend on the fast variable as it will be pointed out further). This is not the case for $\theta$ and an asymptotic smooth limit cannot be considered.

We shall see later that the functions $p_{i}, i \geq 1$, are defined up to an additive constant. Moreover, from Equations (21)-(23), the following properties hold:

$$
p_{0}(x) \geq 0
$$




$$
\begin{gathered}
0 \leq \theta_{0}(x, y) \leq 1, \\
p_{0}(x, y)\left(1-\theta_{0}(x, y)\right)=0 .
\end{gathered}
$$

Putting Equations (7) and (8) into Equation (20), one can write, by an identification procedure, the following macroscopic / microscopic decomposition:

- Macroscopic equation:

$$
\begin{gathered}
\sum_{j=1}^{2} \frac{\partial}{\partial x_{j}}\left\{\overline{h_{\left[p_{0}\right]}^{3}(x, y)} e^{-\alpha p_{0}(x)}\left(\frac{\partial p_{0}}{\partial x_{j}}(x)+\frac{\partial p_{1}}{\partial y_{j}}(x, y)\right)^{Y}\right\} \\
=\frac{\partial}{\partial x_{1}}\left\{\overline{\theta_{0}(x, y) h_{\left[p_{0}\right]}(x, y)} Y\right\}
\end{gathered}
$$

where $\bar{u}^{Y}$ denotes the local average of any $Y$ periodic function $u$ :

$$
\bar{u}^{Y}(x)=\frac{1}{[Y]} \int_{Y} u(x, y) d y .
$$

- Microscopic equation: for each $x$, we have

$$
\begin{aligned}
\sum_{j=1}^{2} \frac{\partial}{\partial y_{j}}\left\{h_{\left[p_{0}\right]}^{3}(x, y)\right. & \left.e^{-\alpha p_{0}(x)}\left(\frac{\partial p_{0}}{\partial x_{j}}(x)+\frac{\partial p_{1}}{\partial y_{j}}(x, y)\right)\right\} \\
& =\frac{\partial}{\partial x_{1}}\left\{\theta_{0}(x, y) h_{\left[p_{0}\right]}(x, y)\right\} .
\end{aligned}
$$

Notice that, in the earlier equation, the variable $x$ only plays the role of a parameter.

Of course, the macroscopic equation (12) is not sufficient to describe a complete asymptotic model: indeed, we have to eliminate $p_{1}$ in order to get a single pressure-saturation equation. For this, we use the microscopic equation (13). Thus we can represent $p_{1}$ as a function of $p_{0}$ and $\theta_{0}$ in a more usable form. We define $w_{\left[p_{0}\right]}^{(i)}, \chi_{\left[p_{0}\right]}^{(i), 0}$ and $\chi_{\left[p_{0}\right]}^{(i, \star)}(i=1,2)$ as the $Y$ periodic solutions (up to an additive constant) of the following local problems:

$$
\begin{aligned}
& \sum_{j=1}^{2} \frac{\partial}{\partial y_{j}}\left(h_{\left[p_{0}\right]}^{3} e^{-\alpha p_{0}} \frac{\partial w_{\left[p_{0}\right]}^{(i)}}{\partial y_{j}}\right)=\frac{\partial h_{\left[p_{0}\right]}^{3}}{\partial y_{i}}, \\
& \sum_{j=1}^{2} \frac{\partial}{\partial y_{j}}\left(h_{\left[p_{0}\right]}^{3} e^{-\alpha p_{0}} \frac{\partial \chi_{\left[p_{0}\right]}^{(i, 0)}}{\partial y_{j}}\right)=\frac{\partial \theta_{0} h_{\left[p_{0}\right]}}{\partial y_{i}},
\end{aligned}
$$




$$
\sum_{j=1}^{2} \frac{\partial}{\partial y_{j}}\left(h_{\left[p_{0}\right]}^{3} e^{-\alpha p_{0}} \frac{\partial \chi_{\left[p_{0}\right]}^{(i, \star)}}{\partial y_{j}}\right)=\frac{\partial h_{\left[p_{0}\right]}}{\partial y_{i}}
$$

Notice that, contrary to the local problems in the purely hydrodynamic case which have been studied in [3], the local problems in the EHL problem highly depend on the macroscopic pressure $p_{0}$, which is due to the piezoviscosity and elastic deformation only.

The solution of Equation (13) reduces to:

$$
\begin{aligned}
p_{1}(x, y)=e^{\alpha p_{0}(x)} \chi_{\left[p_{0}\right]}^{(1,0)}(x, y) & -\frac{\partial p_{0}}{\partial x_{1}}(x) w_{\left[p_{0}\right]}^{(1)}(x, y) \\
& -\frac{\partial p_{0}}{\partial x_{2}}(x) w_{\left[p_{0}\right]}^{(2)}(x, y) .
\end{aligned}
$$

By exchanging the integral and the derivation symbols, and after some calculations, Equation (12) becomes:

$$
\sum_{i, j} \frac{\partial}{\partial x_{i}}\left\{\left(A_{\left[p_{0}\right]}^{\star}\right)_{i j} e^{-\alpha p_{0}} \frac{\partial p_{0}}{\partial x_{j}}\right\}=\sum_{i} \frac{\partial}{\partial x_{i}}\left\{B_{\left[p_{0}\right]}^{(i, 0)}\right\},
$$

where $(i, j=1,2$ and $j \neq i)$

$$
\begin{aligned}
& \left(A_{\left[p_{0}\right]}^{\star}\right)_{i i}={\overline{h_{\left[p_{0}\right]}^{3}-h_{\left[p_{0}\right]}^{3} \frac{\partial w_{\left[p_{0}\right]}^{(i)}}{\partial y_{i}}}}_{Y}^{Y} \\
& \left(A_{\left[p_{0}\right]}^{\star}\right)_{i j}=-h_{\left[p_{0}\right]}^{3} \frac{\partial w_{\left[p_{0}\right]}^{(j)}}{\partial y_{i}}=-\overline{h_{\left[p_{0}\right]}^{3} \frac{\partial w_{\left[p_{0}\right]}^{(i)}}{\partial y_{j}}}=\left(A_{\left[p_{0}\right]}^{\star}\right)_{j i},
\end{aligned}
$$

and also

$$
B_{\left[p_{0}\right]}^{(1,0)}=\overline{\theta_{0} h_{\left[p_{0}\right]}-h_{\left[p_{0}\right]}^{3} \frac{\partial \chi_{\left[p_{0}\right]}^{(1,0)}}{\partial y_{1}}}, \quad B_{\left[p_{0}\right]}^{(2,0)}=-h_{\left[p_{0}\right]}^{3} \frac{\partial \chi_{\left[p_{0}\right]}^{(1,0)}}{\partial y_{2}} \text {. }
$$

In the end, we can also define the normalized coefficients

$$
\begin{aligned}
& B_{\left[p_{0}\right]}^{(1, \star)}=\overline{h_{\left[p_{0}\right]}-h_{\left[p_{0}\right]}^{3} \frac{\partial \chi_{\left[p_{0}\right]}^{(1, \star)}}{\partial y_{1}}}, \quad B_{\left[p_{0}\right]}^{(2, \star)}=-\overline{h_{\left[p_{0}\right]}^{3} \frac{\partial \chi_{\left[p_{0}\right]}^{(1, \star)}}{\partial y_{2}}}, \\
& \Theta_{1}=\frac{B_{\left[p_{0}\right]}^{(1,0)}}{B_{\left[p_{0}\right]}^{(1, \star)}}, \quad \quad \Theta_{2}=\frac{B_{\left[p_{0}\right]}^{(2,0)}}{B_{\left[p_{0}\right]}^{(2, \star)}},
\end{aligned}
$$


which provide the following generalized Reynolds equation:

$$
\sum_{i, j=1}^{2} \frac{\partial}{\partial x_{i}}\left\{\left(A_{\left[p_{0}\right]}^{\star}\right)_{i j} e^{-\alpha p_{0}} \frac{\partial p_{0}}{\partial x_{j}}\right\}=\sum_{i=1}^{2} \frac{\partial}{\partial x_{i}}\left\{\Theta_{i} B_{\left[p_{0}\right]}^{(i, *)}\right\} .
$$

Moreover it is obvious that the following properties hold:

$$
p_{0} \geq 0, \quad p_{0}\left(1-\Theta_{i}\right)=0, \quad(i=1,2) .
$$

Equations (18) and (19) deal with any periodic roughness pattern. To be noticed is the fact that the differential operator is no more of the Reynolds type since extra terms $\partial^{2} p_{0} / \partial x_{i} \partial x_{j}$ appear. The right-hand side also contains an additive term in the $x_{2}$ direction. However, the link between $p_{0}$ and $\Theta_{i}$ is not so clear. This is a major obstacle which prevents to get a tractable equation. In fact, the pressure / double saturation problem lacks some properties: we cannot prove that the so-called anisotropic saturations $\Theta_{i}$ are functions with values in $[0,1]$. However, it is possible to define in a rigorous way (see [2]) a solution with an isotropic saturation $\Theta=\Theta_{1}=\Theta_{2}$ and $0 \leq \Theta \leq 1$. Moreover, under some additional assumptions (see [3]), we can prove some complementary results. Thus, when dealing with transverse roughness and longitudinal roughness, the homogenized coefficients can be easily simplified and given in an explicit form. In both cases, the asymptotic system has the same structure than the initial (roughless) one, i.e.

$$
\begin{gathered}
\sum_{i=1}^{2} \frac{\partial}{\partial x_{i}}\left\{A_{\left[p_{0}\right]}^{(i, \star)} e^{-\alpha p_{0}} \frac{\partial p_{0}}{\partial x_{i}}\right\}=\frac{\partial \Theta B_{\left[p_{0}\right]}^{(1, \star)}}{\partial x_{1}}, \\
p_{0} \geq 0 \\
0 \leq \Theta \leq 1 \\
p_{0}(1-\Theta)=0 .
\end{gathered}
$$

$\triangleright$ Transverse roughness: the homogenized coefficients are

$$
A_{\left[p_{0}\right]}^{(1, \star)}=\frac{1}{\overline{h_{\left[p_{0}\right]}^{-3}} Y}, \quad A_{\left[p_{0}\right]}^{(2, \star)}={\overline{h_{\left[p_{0}\right]}^{3}}}^{3}, \quad B_{\left[p_{0}\right]}^{(1, \star)}=\frac{{\overline{h_{\left[p_{0}\right]}^{-2}}}^{\bar{h}_{\left[p_{0}\right]}^{-3}}}{},
$$

the link between the macroscopic saturation $\Theta$ and the microscopic one $\theta_{0}$ being given by:

$$
\Theta(x)=\left(\frac{1}{{\overline{h_{\left[p_{0}\right]}^{-2}}}^{Y}} \overline{\left(\frac{\theta_{0}}{h_{\left[p_{0}\right]}^{2}}\right)}\right)(x) .
$$


$\triangleright$ Longitudinal roughness: the homogenized coefficients are

$$
A_{\left[p_{0}\right]}^{(1, \star)}={\overline{h_{\left[p_{0}\right]}^{3}}}^{Y}, \quad A_{\left[p_{0}\right]}^{(2, \star)}=\frac{1}{\overline{h_{\left[p_{0}\right]}^{-3} Y}}, \quad B_{\left[p_{0}\right]}^{(1, \star)}=\overline{h_{\left[p_{0}\right]}} Y
$$

the link between the macroscopic saturation $\Theta$ and the microscopic one $\theta_{0}$ being given by:

$$
\Theta(x)=\left(\frac{\overline{\theta_{0} h_{\left[p_{0}\right]}}}{\overline{h_{\left[p_{0}\right]}} Y}\right)(x) .
$$

All the earlier results are valid for both elastohydrodynamic and hydrodynamic cases and, thus, generalize the ones that have been stated in [3]. As an important feature, $\Theta$ is not the average of the microscopic saturation $\theta_{0}$ but contains some anisotropic effects due to the roughness direction. In the purely hydrodynamic case, one can prove some additional results, corresponding to a wide class of two dimensional roughness patterns. Indeed, suppose that $h_{r}$ can be written under the form

$$
h_{r}(x, y)=h_{1}\left(x, y_{1}\right) h_{2}\left(x, y_{2}\right)
$$

then we get the following (hydrodynamic) homogenized equation

$$
\begin{aligned}
& \sum_{i=1}^{2} \frac{\partial}{\partial x_{i}}\left\{A^{(i, \star)} \frac{\partial p_{0}}{\partial x_{i}}\right\}=\frac{\partial}{\partial x_{1}}\left\{\Theta B^{(1, \star)}\right\} \\
& p_{0} \geq 0, \quad 0 \leq \Theta \leq 1, \quad p_{0}(1-\Theta)=0
\end{aligned}
$$

with

$$
A^{(i, \star)}=\frac{{\overline{h_{j}^{3}}}^{Y}}{{\overline{h_{i}^{-3}}}^{Y}}, \quad B^{(1, \star)}=\frac{{\overline{h_{1}^{-2}}}^{Y}}{{\overline{h_{1}^{-3}}}^{Y}}{\overline{h_{2}}}^{Y},
$$

the link between the macroscopic and the microscopic saturations being given by

$$
\Theta=\frac{1}{{\overline{h_{2}}}^{Y} \overline{h_{1}^{-2}} Y} \overline{\left(\frac{\theta_{0} h_{2}}{h_{1}^{2}}\right)} Y
$$




\section{Numerical results}

In this section, the numerical simulation of a micro(elasto)hydrodynamic contact is performed to illustrate the theoretical results of convergence stated in the previous sections. For the numerical solution of the $\varepsilon$ dependent problems and their corresponding homogenized one, we propose the characteristics method adapted to steady state problems to deal with the the convection term combined with a finite element spatial discretization. These numerical techniques has been already successfully used in previous papers dealing with hydrodynamic aspects (see $[8,9]$ ), and elastohydrodynamic aspects (see, for instance, $[10,11])$.

In TABLE 1 , we present the functional coefficients $A_{\left[p_{0}\right]}^{(1, \star)}, A_{\left[p_{0}\right]}^{(2, \star)}, B_{\left[p_{0}\right]}^{(1, \star)}$ that appear in the homogenized problem for purely transverse and purely longitudinal roughness cases which have been partially computed with MATHEMATICA Software Package. Suppose that the domain to be considered is a rectangular one

$$
(a, b) \times(c, d),
$$

and that the rough (elasto)hydrodynamic gap can be written under the form

$h_{[p]}\left(x, \frac{x}{\varepsilon}\right)=h_{r}(x)+h_{d}[p](x)+ \begin{cases}\alpha_{1} \sin \left(\frac{2 \pi}{\varepsilon} \frac{x_{1}-a}{b-a}\right), & \text { (transverse roughness) } \\ \alpha_{2} \sin \left(\frac{2 \pi}{\varepsilon} \frac{x_{2}-c}{d-c}\right), & \text { (longitudinal roughness) }\end{cases}$

where $h_{r}$ (resp. $\left.h_{d}[p]\right)$ denotes the roughless rigid (resp. elastic) contribution to the gap and the remaining term describes the roughness patterns in the purely transverse or longitudinal case.

\begin{tabular}{|c||c|c|}
\hline & Transverse roughness & Longitudinal roughness \\
\hline \hline$h_{[p]}(x, y)$ & $h_{r}(x)+h_{d}[p](x)+\alpha_{1} \sin \left(2 \pi y_{1}\right)$ & $h_{r}(x)+h_{d}[p](x)+\alpha_{2} \sin \left(2 \pi y_{2}\right)$ \\
\hline$A_{\left[p_{0}\right]}^{(1, \star)}$ & $2 \frac{\left(\left(h_{r}+h_{d}[p]\right)^{2}-\alpha_{1}^{2}\right)^{5 / 2}}{2\left(h_{r}+h_{d}[p]\right)^{2}+h_{r}^{2}}$ & $\left(h_{r}+h_{d}[p]\right)^{3}+\frac{3}{2}\left(h_{r}+h_{d}[p]\right) \alpha_{2}^{2}$ \\
\hline$A_{\left[p_{0}\right]}^{(2, \star)}$ & $\left(h_{r}+h_{d}[p]\right)^{3}+\frac{3}{2}\left(h_{r}+h_{d}[p]\right) \alpha_{1}^{2}$ & $2 \frac{\left(\left(h_{r}+h_{d}[p]\right)^{2}-\alpha_{2}^{2}\right)^{5 / 2}}{2\left(h_{r}+h_{d}[p]\right)^{2}+\alpha_{2}^{2}}$ \\
\hline$B_{\left[p_{0}\right]}^{(1, \star)}$ & $2\left(h_{r}+h_{d}[p]\right) \frac{\left(h_{r}+h_{d}[p]\right)^{2}-\alpha_{1}^{2}}{2\left(h_{r}+h_{d}[p]\right)^{2}+\alpha_{1}^{2}}$ & $h_{r}+h_{d}[p]$ \\
\hline
\end{tabular}

Table 1

Elastohydrodynamic homogenized coefficients 


\subsection{Hydrodynamic case}

We adress the numerical simulation of journal bearing devices with circumferential supply of lubricant. The mechanical characteristics of the device are given by:

- length: $L=0.019 \mathrm{~m}$,

- bearing radius: $R_{b}=0.0164975 \mathrm{~m}$,

- journal radius: $R_{j}=0.01647 \mathrm{~m}$,

- mean radius: $R_{m}=0.5\left(R_{b}+R_{j}\right)$,

- clearance : $c=R_{b}-R_{j}$,

- eccentricity: $\rho=0.2$.

The physical characteristics of the regime are the following one:

- supply pressure: $p_{a}=283000 P a$,

- lubricant viscosity: $\mu=0.02$ Pa.s,

- shear velocity: $v_{0}=17.247 \mathrm{~m} / \mathrm{s}$.

The earlier problem leads to the following set of equations

$$
\begin{aligned}
& \sum_{i=1}^{2} \frac{\partial}{\partial x_{i}}\left(h^{3} \frac{\partial p}{\partial x_{i}}\right)=\Lambda \frac{\partial \theta h}{\partial x_{1}}, \\
& p \geq 0, \quad 0 \leq \theta \leq 1, \quad p(1-\theta)=0,
\end{aligned}
$$

with $\Lambda=6 \mu v_{0}$, and the real roughless gap shoud be read as

$$
h(x)=c\left(1+\rho \cos \left(x_{1} / R_{m}\right)\right) .
$$

The equations have to be solved on the domain $\left(0,2 \pi R_{m}\right) \times(0, L / 2)$ (see the developped configuration on FIG.2) with the following boundary conditions:

- $p=p_{a}$ on $\Gamma_{a}, p=0$ on $\Gamma_{0}$,

- periodic conditions on $\Gamma_{\sharp}$, i.e. $p$ and $6 \mu v_{0} \theta h-h^{3} \frac{\partial p}{\partial x_{1}}$ are $2 \pi R_{m} x_{1}$ periodic.

To be noticed is the fact that the corresponding homogenized boundary conditions can be written as follows (using the terminology of TABLE 1):

- $p_{0}=p_{a}$ on $\Gamma_{a}, p_{0}=0$ on $\Gamma_{0}$,

- periodic conditions on $\Gamma_{\sharp}$, i.e. $p_{0}$ and $6 \mu v_{0} \Theta B^{(1, \star)}-A^{(1, \star)} \frac{\partial p_{0}}{\partial x_{1}}$ are $2 \pi R_{m} x_{1}$ periodic. 


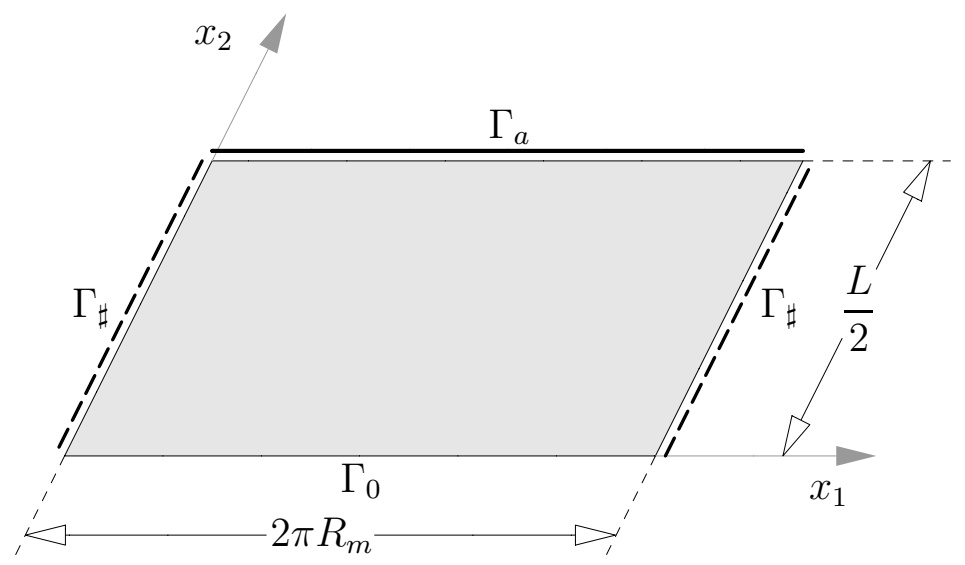

Fig. 2. Journal bearing domain

Actually, we consider transverse roughness patterns and the gap should be read as:

$$
h\left(x, \frac{x}{\varepsilon}\right)=c\left(1+\rho \cos \left(x_{1} / R_{m}\right)+a_{r} \sin \left(\frac{2 \pi}{\varepsilon} \frac{x_{1}}{R_{m}}\right)\right)
$$

with $a_{r} / \rho=0.2, a_{r}$ denoting the amplitude parameter of the roughness.

FIG.3 and 4 (resp. 5 and 6) show the pressure and saturation profiles for $\varepsilon=1 / 15$ (resp. $\varepsilon=1 / 30$ ) compared to the homogenized solution, at a fixed $x_{2}^{0}=L / 4$. Thus, it allows to observe the roughness effects in the $x_{1}$ direction. The amplitude of the pressure oscillations tend to be damped, although the amplitude of the saturation oscillations stay the same in cavitated areas. As it was noticed in [3], it points out the fact that when the number of roughness patterns increases, the pressure behaves as a smooth function, namely $p_{0}(x)$, while the saturation behaves as a highly oscillating function, namely $\theta_{0}(x, x / \varepsilon)$. Thus, the pressure tends to a smooth one as $\varepsilon$ tends to 0 , while the saturation is always oscillating. To be noticed on FIG. 4 and 6 is the fact that the cavitation area is made of two macrocavitation zones (for $\varepsilon=1 / 15, x_{1}>0.06$ and $\left.x_{1}<0.01\right)$ and a lot of microcavitation zones.

Fig.7 and 8 represent the homogenized pressure and saturation in the real domain.

\subsection{Elastohydrodyamic case}

The numerical tests deal with a dimensionless problem, as described in Section 2 : the domain is $(-4,2) \times(2,2)$. The considered rigid contribution to the gap is a normalized one:

$$
h_{0}+\frac{x_{1}^{2}+x_{2}^{2}}{2}
$$




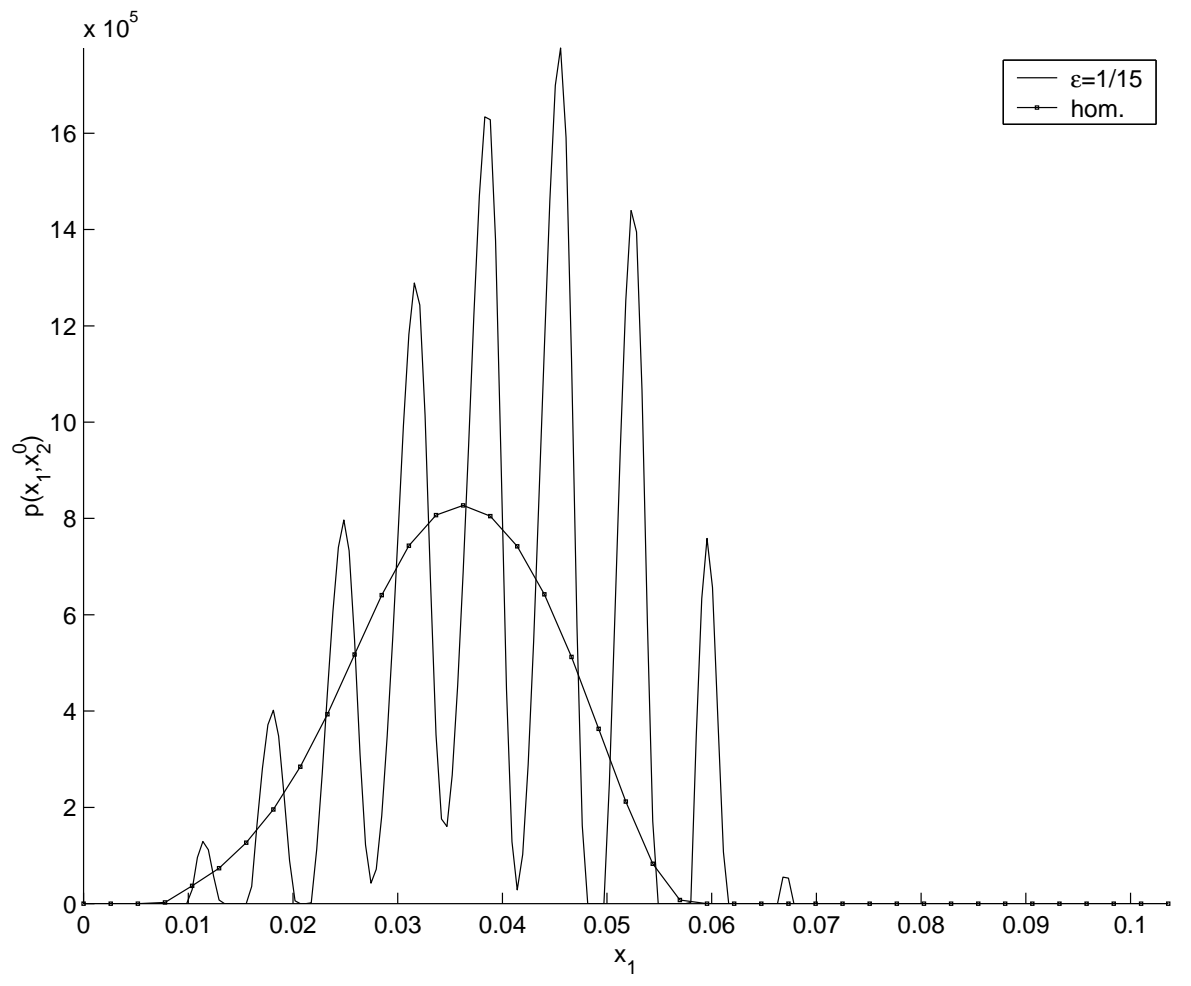

Fig. 3. Hydrodynamic pressure for $\varepsilon=1 / 15$ at $x_{2}^{0}=L / 4$

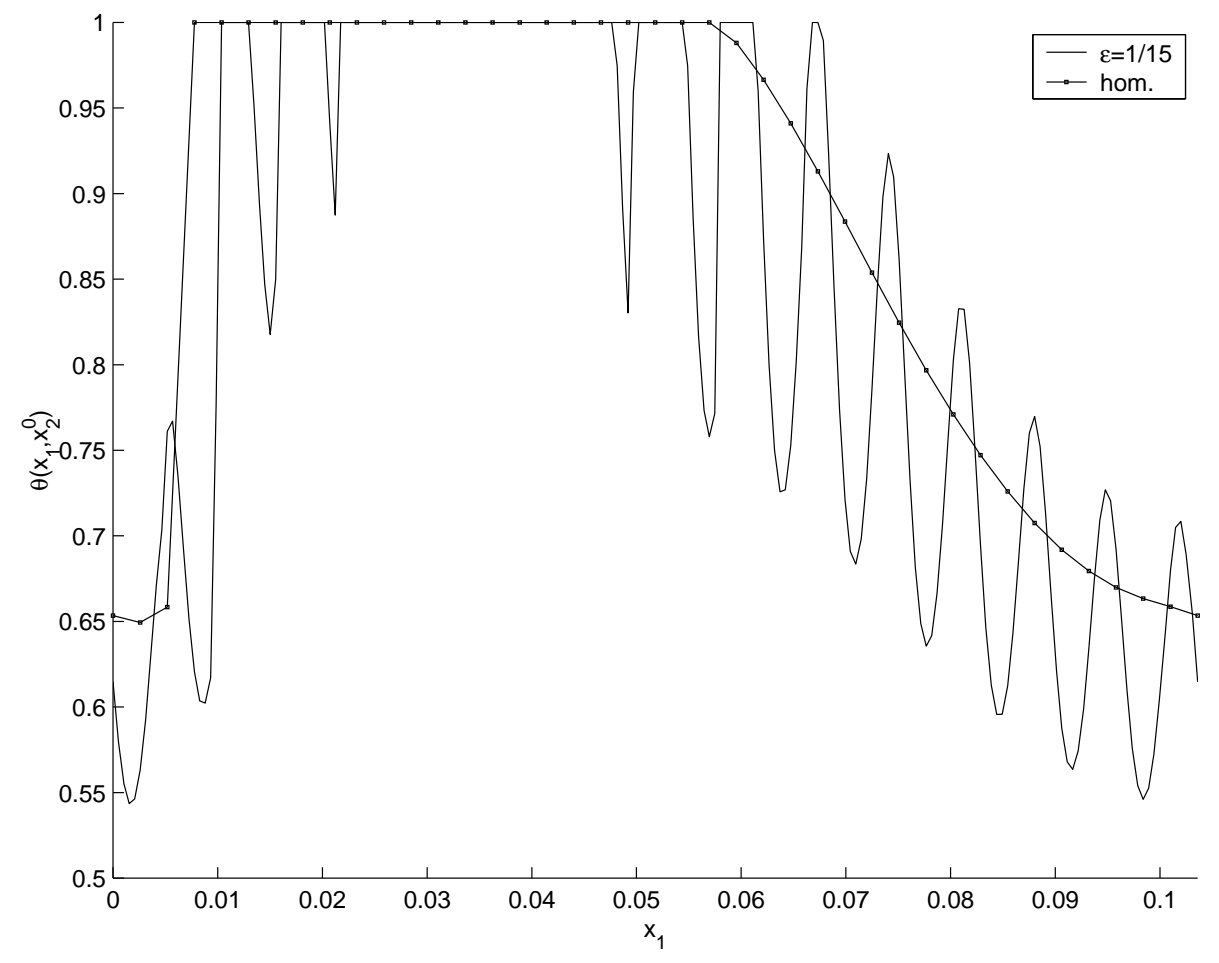

Fig. 4. Hydrodynamic saturation for $\varepsilon=1 / 15$ at $x_{2}^{0}=L / 4$ 


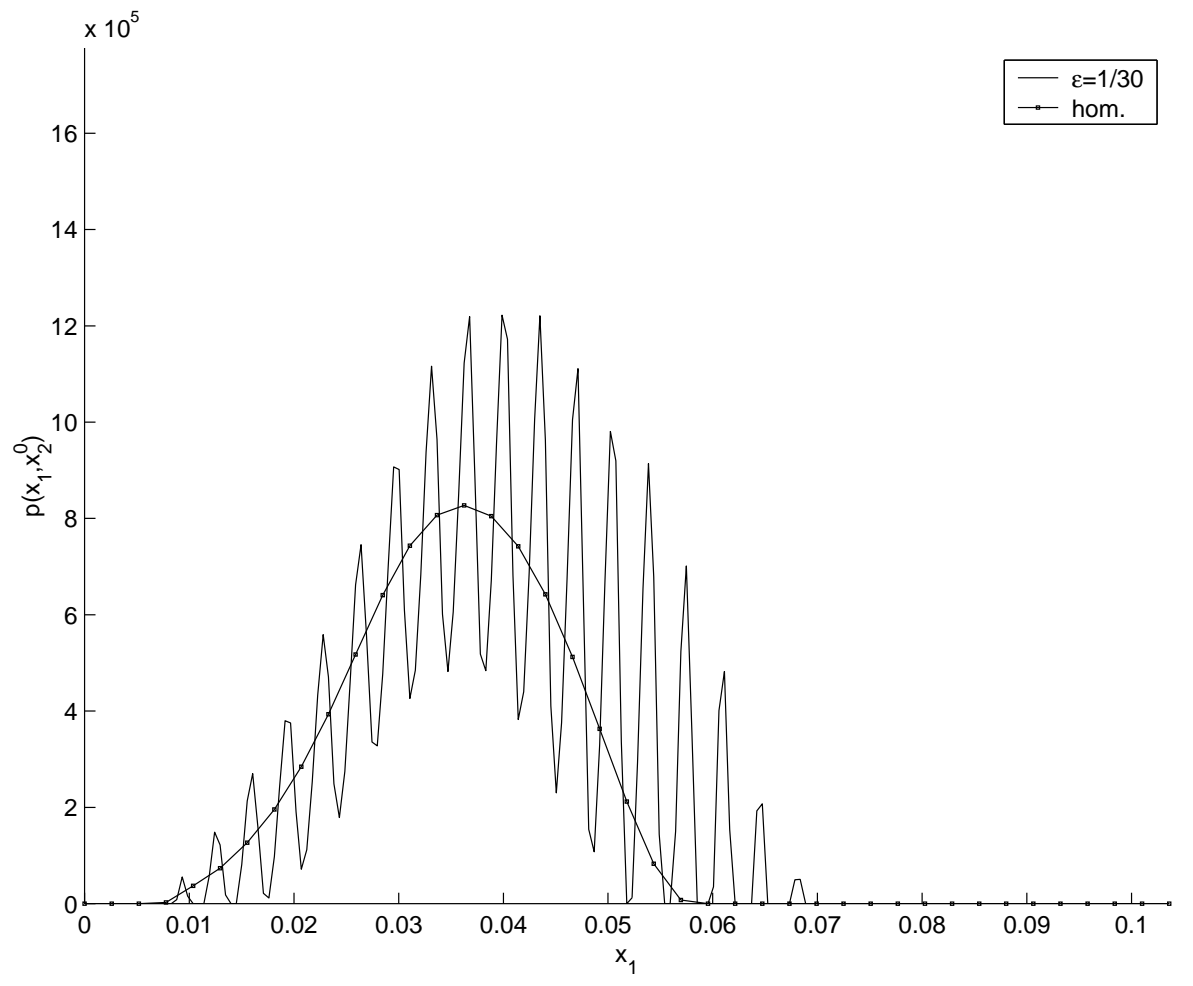

Fig. 5. Hydrodynamic pressure for $\varepsilon=1 / 30$ at $x_{2}^{0}=L / 4$

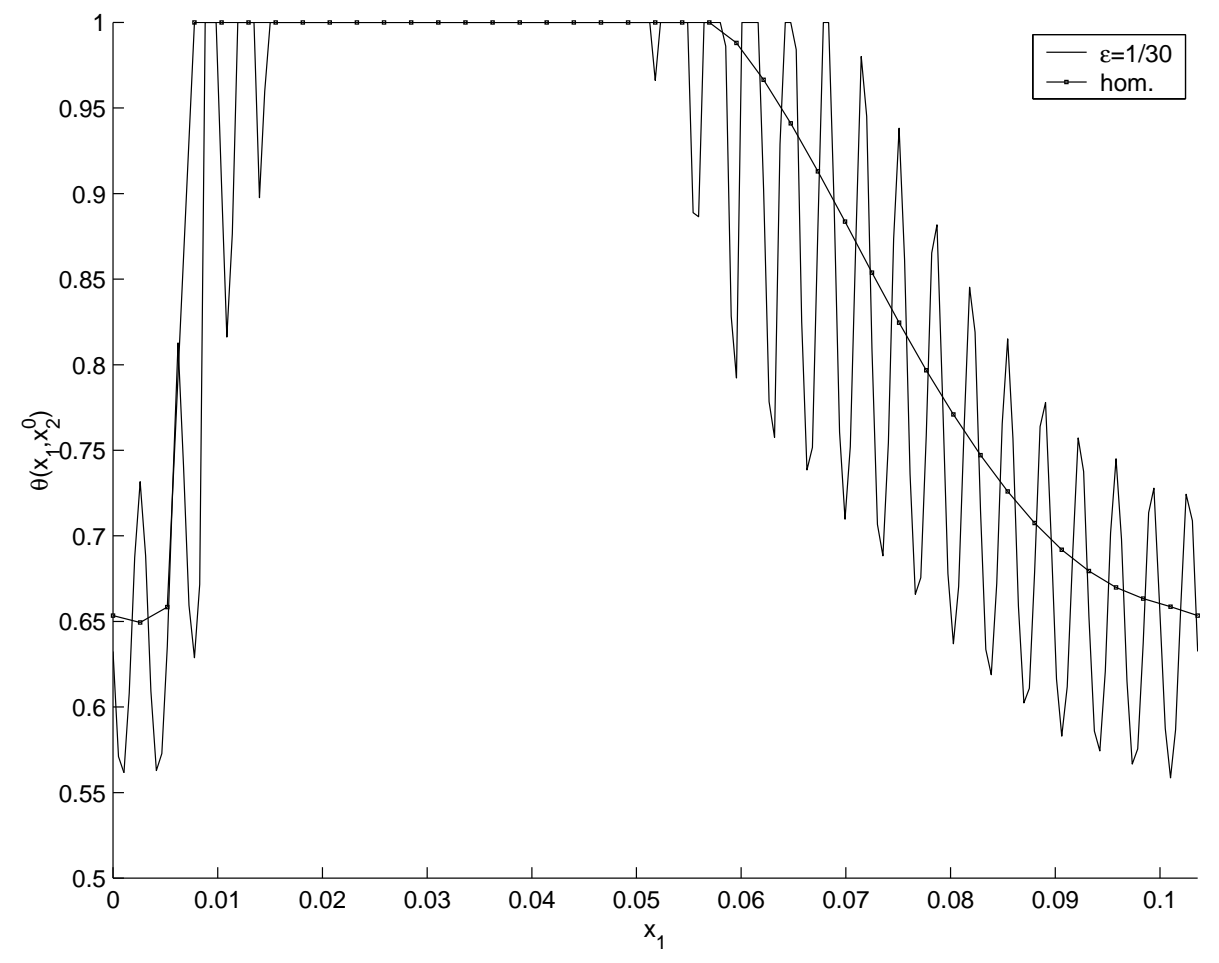

Fig. 6. Hydrodynamic saturation for $\varepsilon=1 / 30$ at $x_{2}^{0}=L / 4$ 


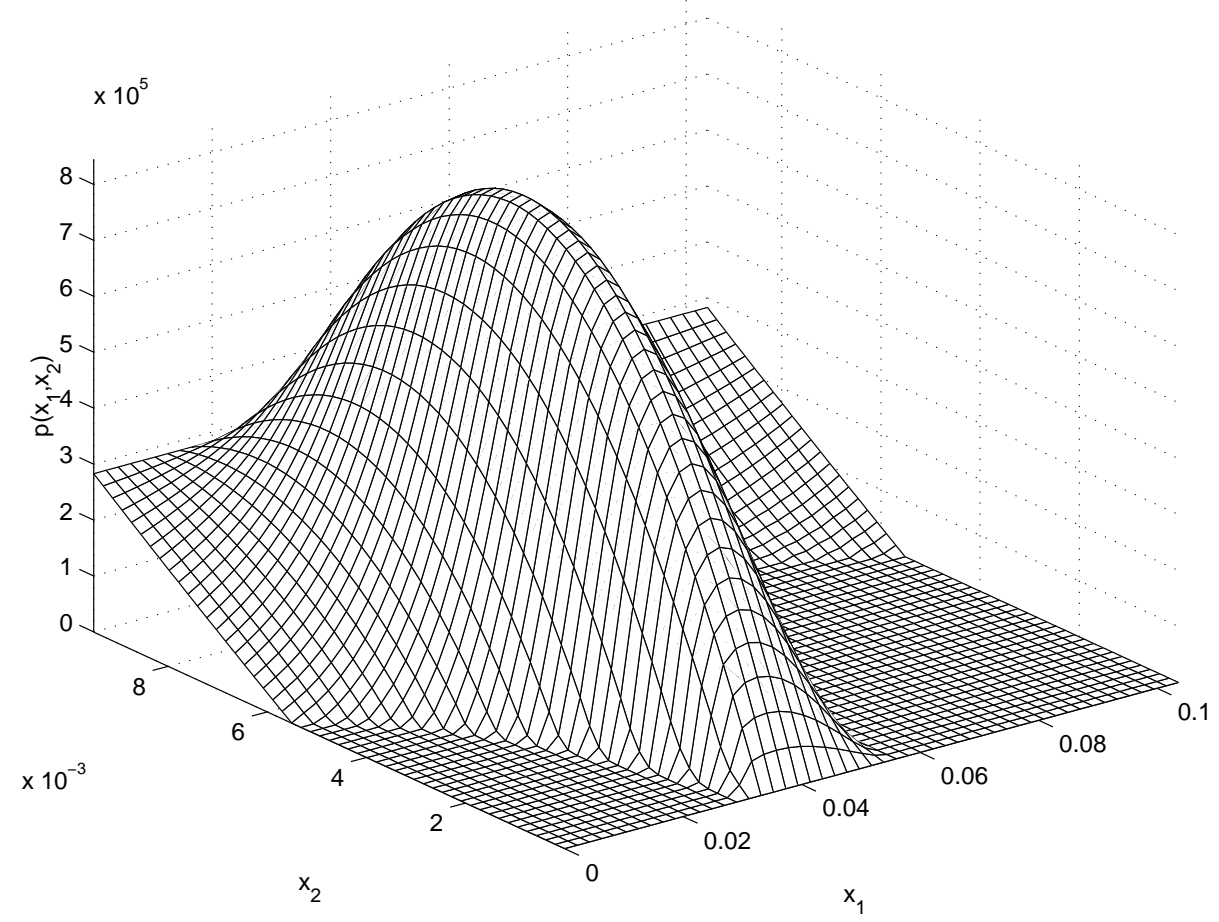

Fig. 7. Homogenized hydrodynamic pressure in the whole device

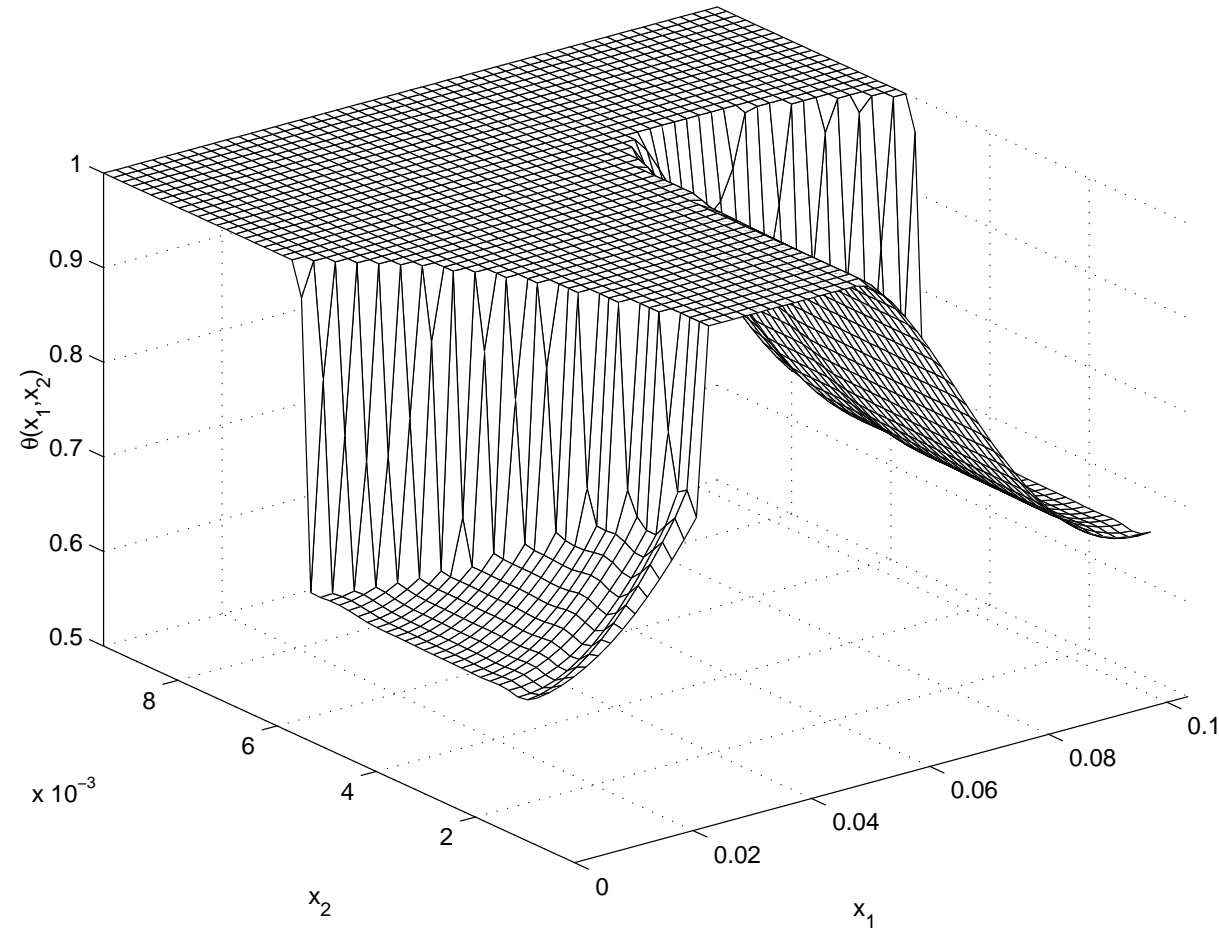

Fig. 8. Homogenized hydrodynamic saturation in the whole device 
where $h_{0}$ denotes the minimum thickness. Since a point contact has been considered, we choose the following Hertz model:

$$
k(x, z)=\frac{2}{\pi^{2}} \frac{1}{\sqrt{\left(x_{1}-z_{1}\right)^{2}+\left(x_{2}-z_{2}\right)^{2}}}
$$

The equations are:

$$
\begin{gathered}
\sum_{i=1}^{2} \frac{\partial}{\partial x_{i}}\left(h[p]^{3} e^{-\alpha p} \frac{\partial p}{\partial x_{i}}\right)=\frac{\partial \theta h[p]}{\partial x_{1}}, \\
p \geq 0, \quad p(1-\theta)=0 \quad 0 \leq \theta \leq 1,
\end{gathered}
$$

The boundary conditions are the following ones:

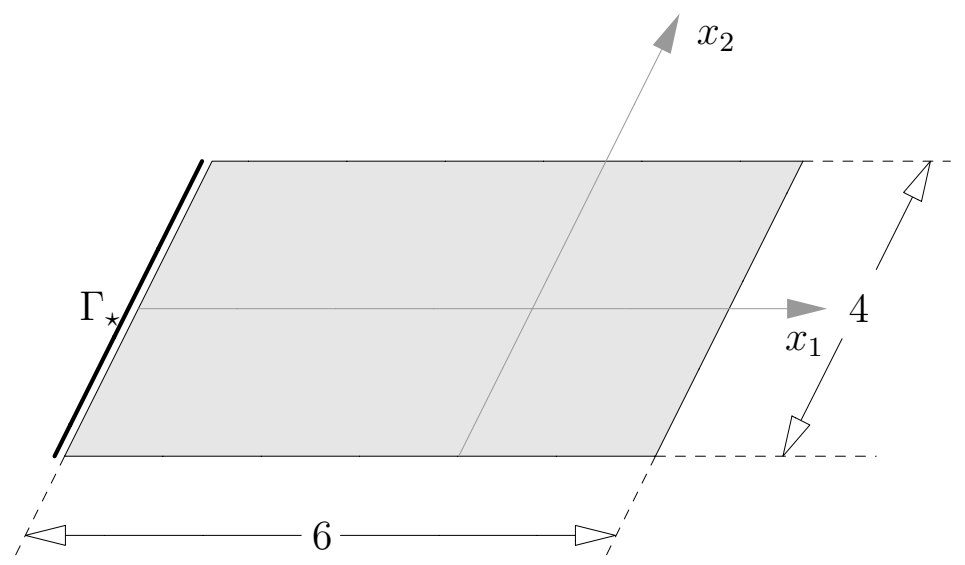

Fig. 9. Normalized EHL domain

- flow condition on $\Gamma_{\star}: \theta h[p]-h[p]^{3} \frac{\partial p}{\partial x_{1}}=\theta_{i n} h[p]$, with $\theta_{i n}=0.3$,

- $p=0$ elsewhere.

The corresponding homogenized boundary conditions can be written as:

- flow condition on $\Gamma_{\star}: \Theta B_{\left[p_{0}\right]}^{(1, \star)}-A_{\left[p_{0}\right]}^{(1, \star)} \frac{\partial p_{0}}{\partial x_{1}}=\theta_{i n} C_{\left[p_{0}\right]}^{(\star)}$, with

$$
C_{\left[p_{0}\right]}^{(\star)}=\left(h_{r}+h_{d}[p]\right)_{\mid \Gamma_{\star}} .
$$

- $p_{0}=0$ elsewhere.

The chosen values of $h_{0}$ and $\alpha$ will be discussed further. 


\subsubsection{Transverse roughness}

Numerical tests have been made for the following rigid contribution to the gap:

$$
h_{0}+\frac{x_{1}^{2}+x_{2}^{2}}{2}+h_{0} \sin \left(2 \pi \frac{x_{1}+4}{6 \varepsilon}\right)
$$

with $h_{0}=0.5$ and different values of $\varepsilon$. Moreover, the piezoviscosity has been taken to $\alpha=1$. Homogenized coefficients in the transverse roughness cases are deduced from TABLE 1 (see page 10).

Fig.10, 12 and 14 represent the pressure, saturation and deformation profiles at $x_{2}^{0}=0$ in the roughless case $(\varepsilon=+\infty)$, a deterministic rough case $(\varepsilon=$ $1 / 30$ ) and the homogenized case. To be observed is the fact that the homogenized profiles give a satisfying approach of the roughness effects $(\varepsilon=1 / 30)$, unlike the roughless profiles: indeed, the pressure profiles given in FIG.10 evidence the fact that the homogenized pressure is a smooth version of the rough pressure. Similarly, by FIG.12, the homogenized saturation can be seen as an average version of the rough saturation, up to anisotropic effects. On FIG.14, we observe that the rough deformation (corresponding to $\varepsilon=1 / 30$ ) nearly coincides with the homogenized one: this is due to the regularizing effects of the Hertz kernel. In fact, the deformation profile has a rate of convergence which is much greater than the pressure profile.

Fig.11, 13 and 15 represent the homogenized pressure, saturation and deformation in the domain.

\subsubsection{Longitudinal roughness}

Numerical tests have been made for the following rigid contribution to the gap:

$$
h_{0}+\frac{x_{1}^{2}+x_{2}^{2}}{2}+h_{0} \sin \left(2 \pi \frac{x_{2}+2}{4 \varepsilon}\right)
$$

with $h_{0}=0.5$ and different values of $\varepsilon$. Moreover, the piezoviscosity has been taken to $\alpha=1$. Let us notice that, again, Homogenized coefficients in the transverse roughness cases are deduced from TABLE 1 (see page 10).

FIG.16 and 17 represent the pressure and deformation profiles at $x_{1}^{0}=-0.4$, in the $x_{2}$ direction (in order to observe the roughness effects). This choice corresponds to the maximum pressure in the homogenized case, which is attained at $\left(x_{1}^{0}, x_{2}^{0}\right)=(-0.4,0)$. Of course, the saturation profile is omitted, for all corresponding saturation functions would be identically equal to 1 (no cavitation in this part of the domain). Significantly, the size of the oscillations for the pressure are damped easily, and convergence of the rough solution to the homogenized one is illustrated on both figures. Similarly, pressure / saturation / deformation curves are omitted, for they are similar to the ones observed in the transverse roughness case. 


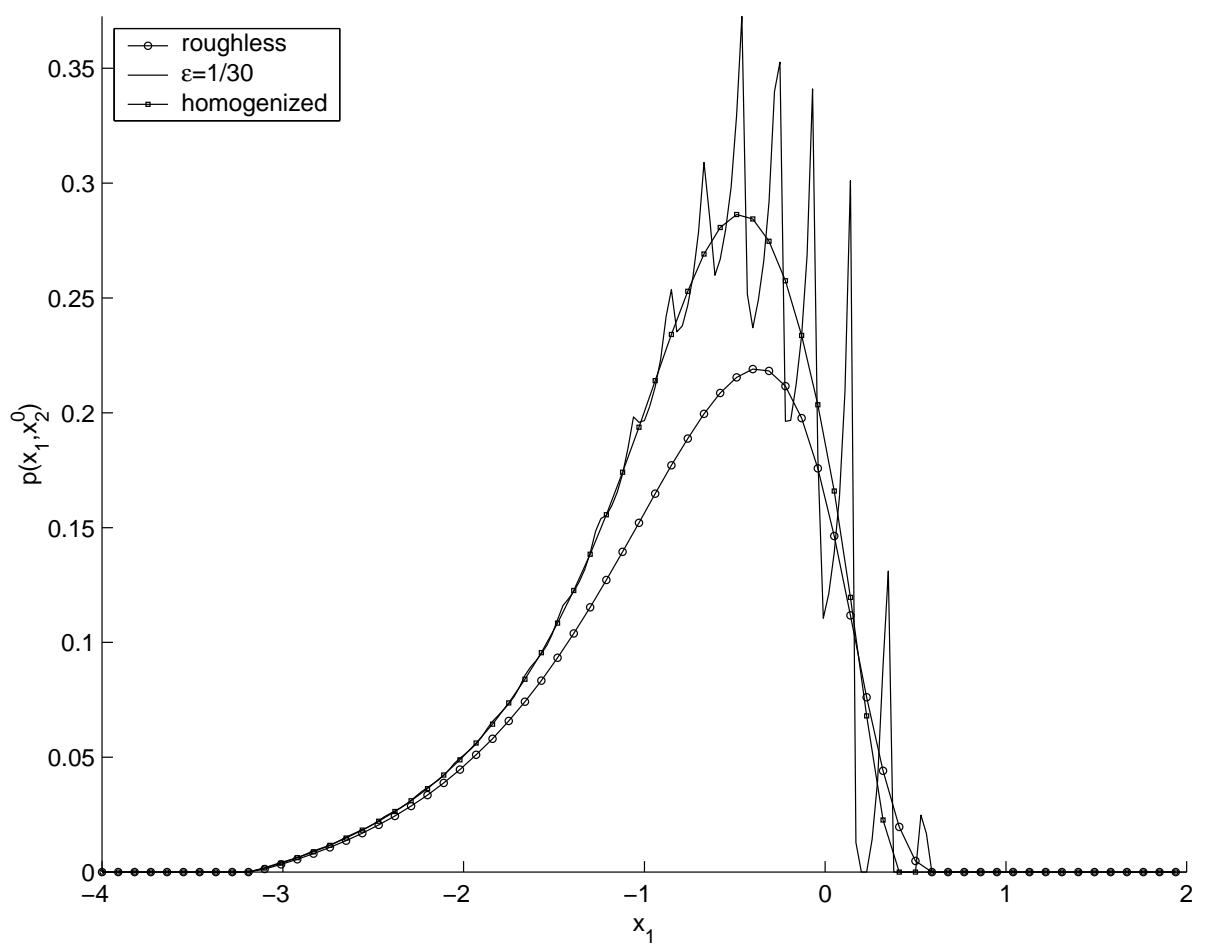

Fig. 10. EHL pressure with transverse roughness patterns at $x_{2}^{0}=0$

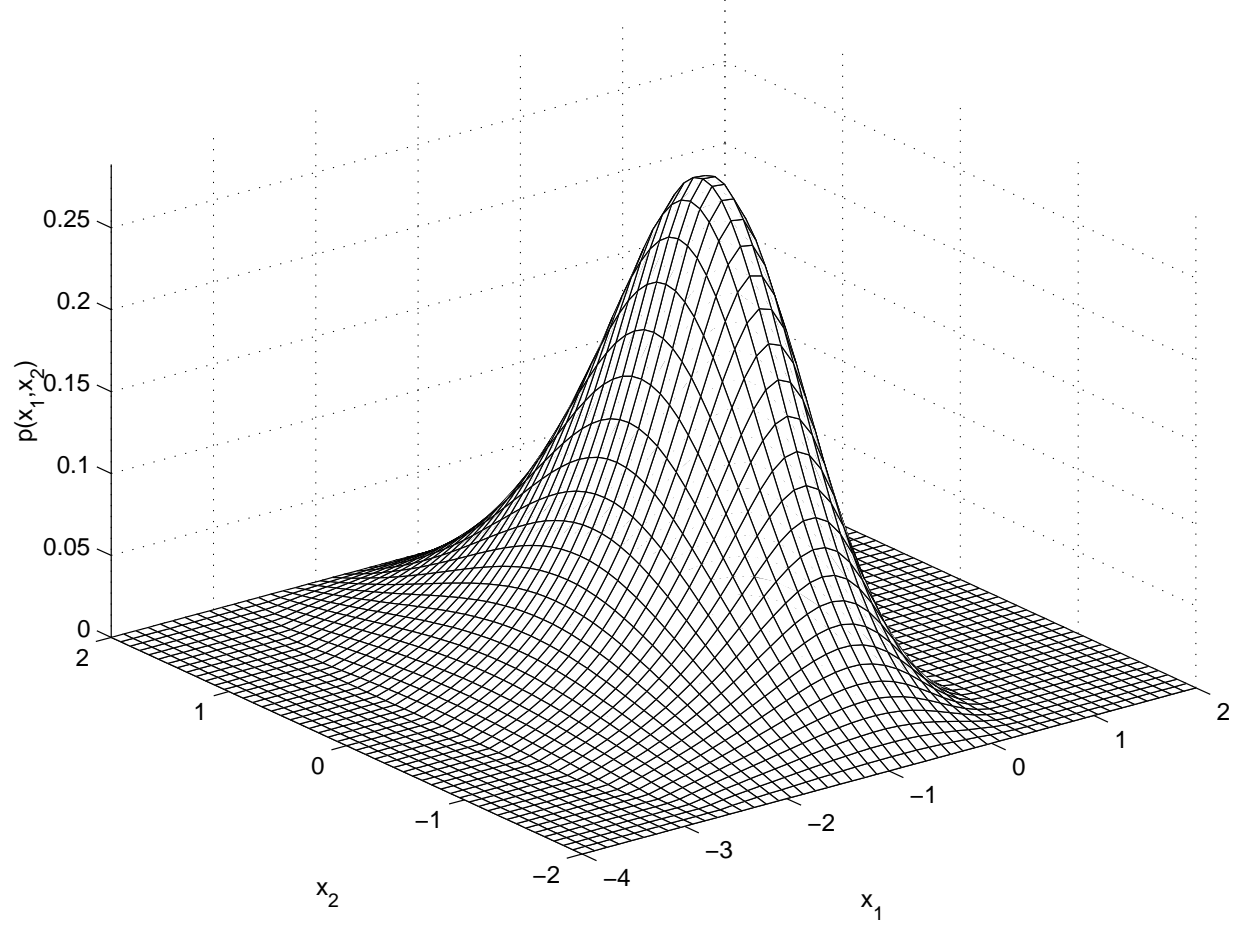

Fig. 11. Homogenized EHL pressure in the whole domain 


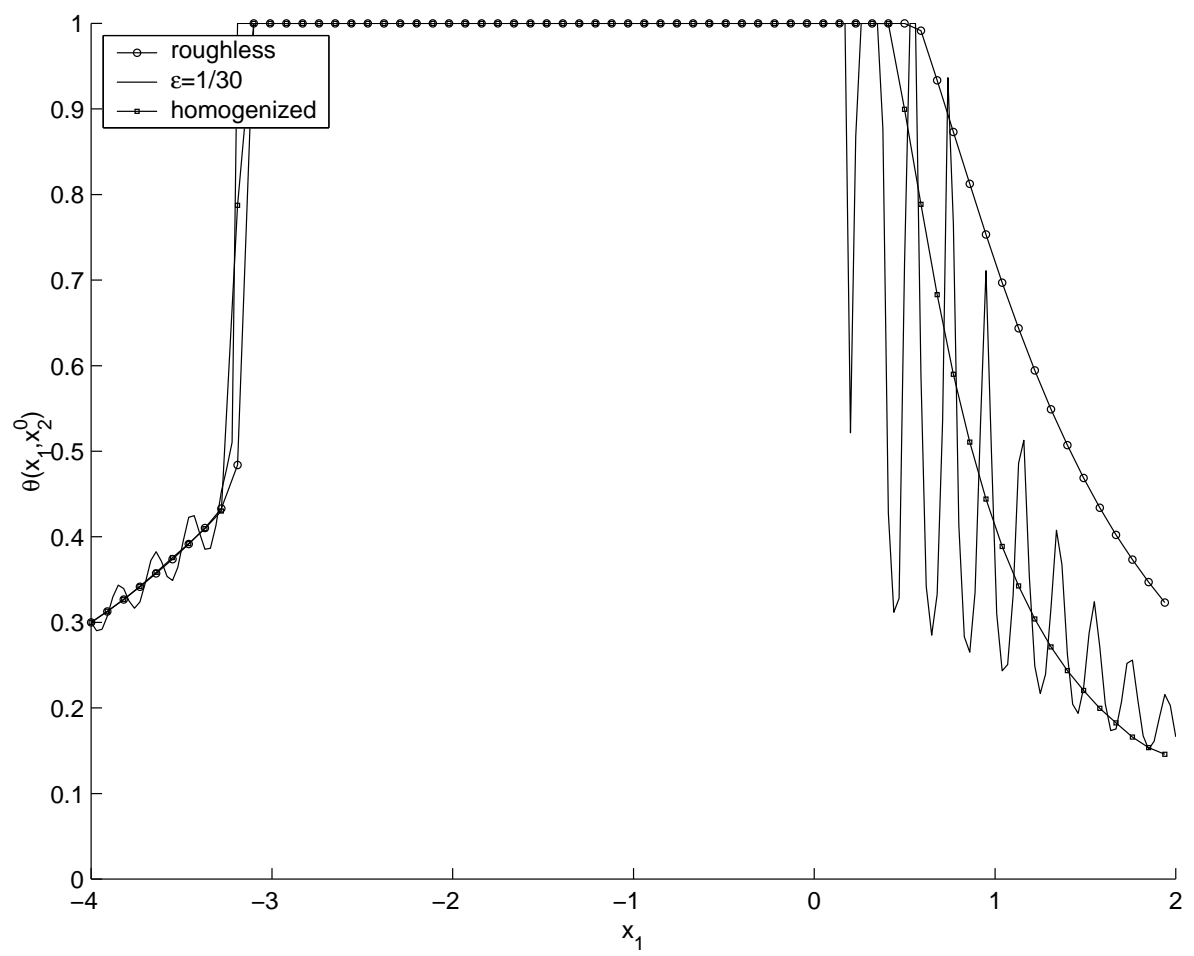

Fig. 12. EHL saturation with transverse roughness patterns at $x_{2}^{0}=0$

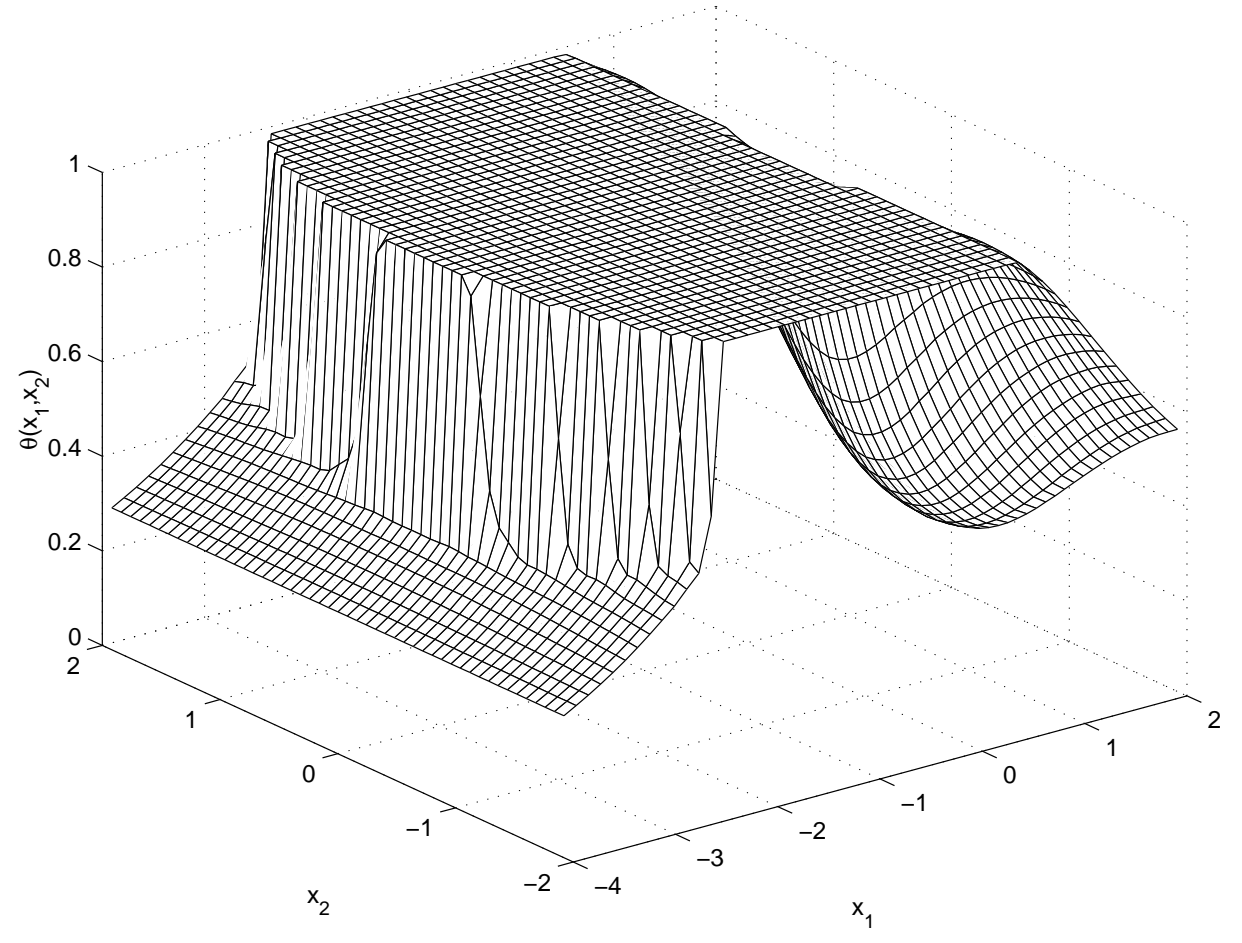

Fig. 13. Homogenized EHL saturation in the whole domain 


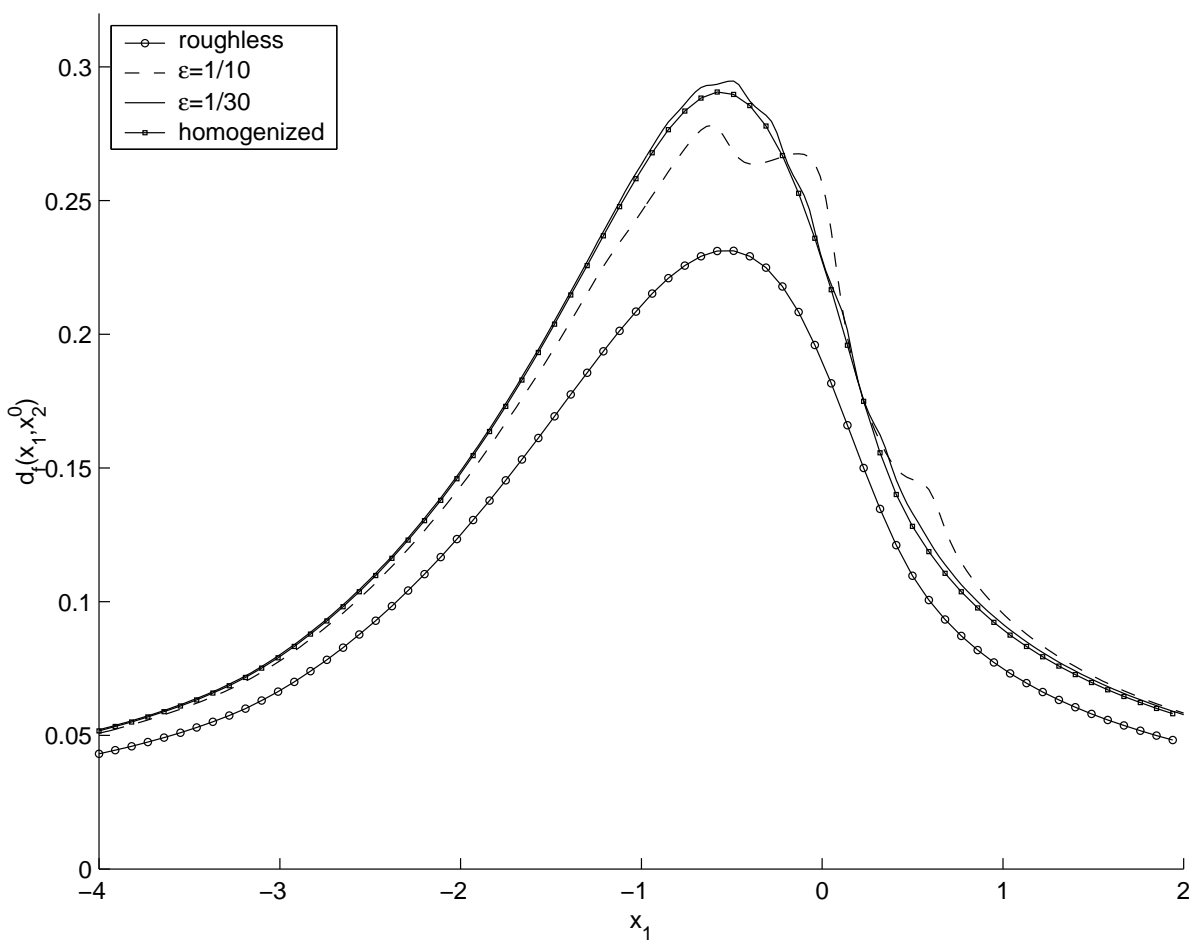

Fig. 14. EHL deformation with transverse roughness patterns at $x_{2}^{0}=0$

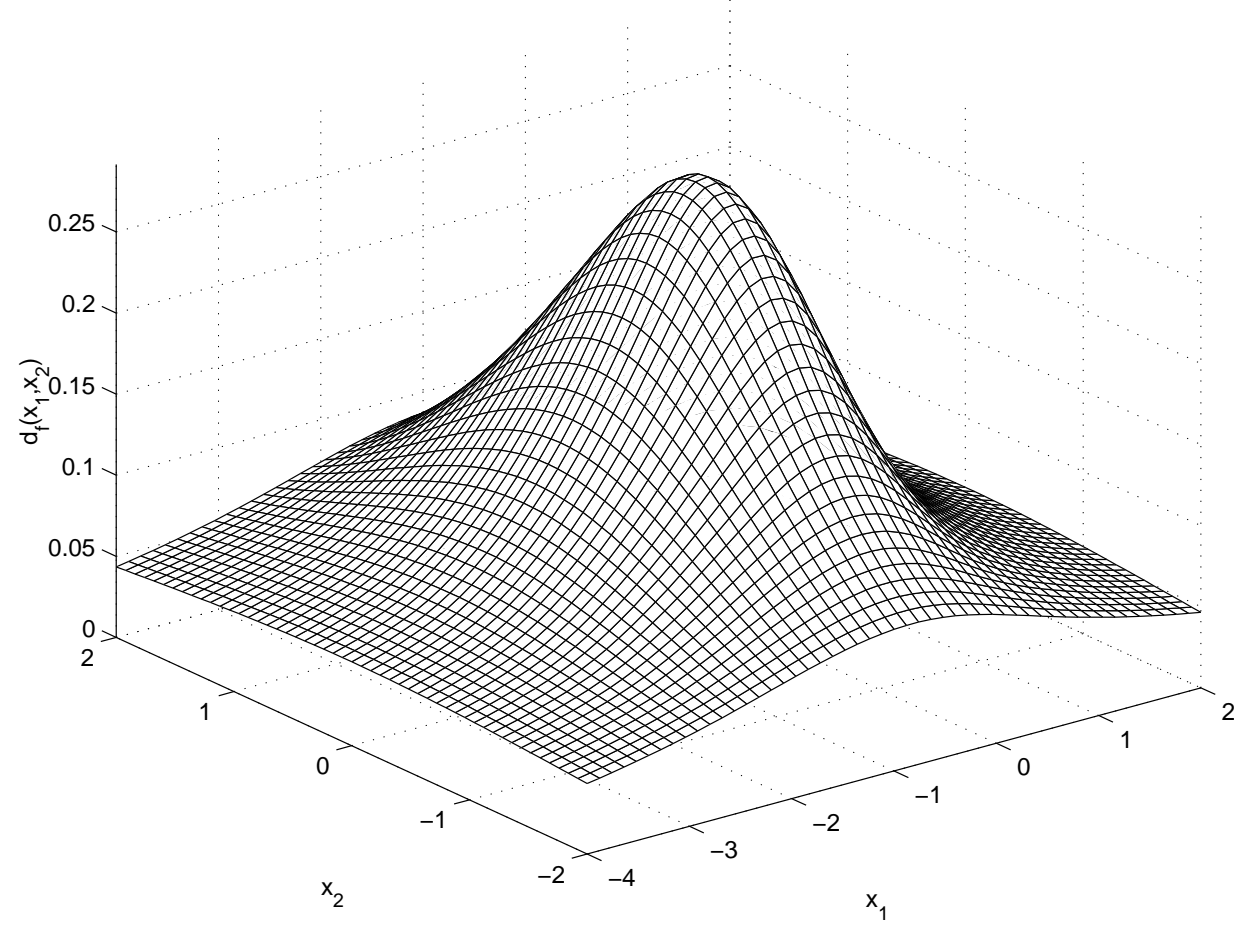

Fig. 15. Homogenized EHL deformation in the whole domain 


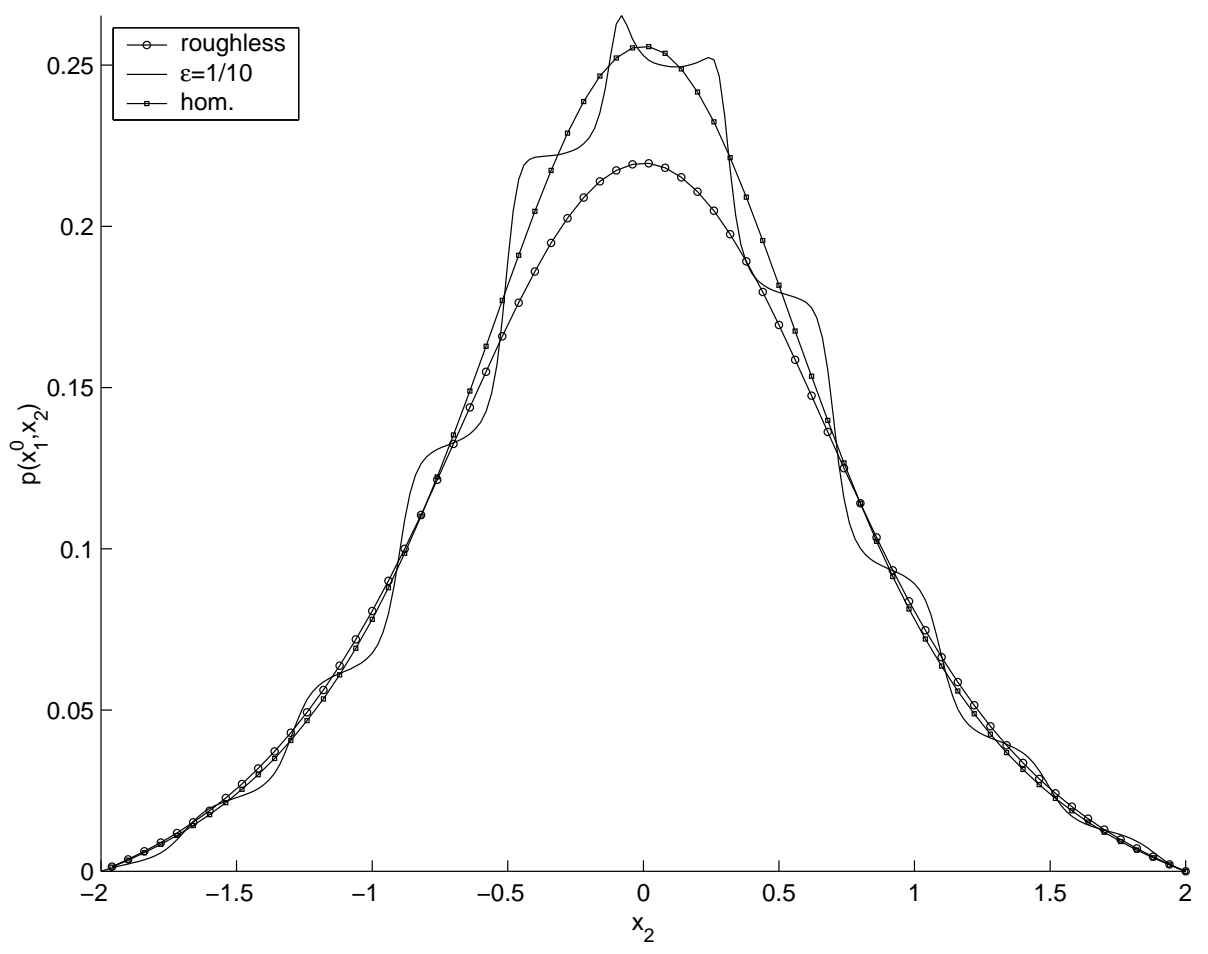

Fig. 16. EHL pressure with longitudinal roughness patterns at $x_{1}^{0}=-0.4$

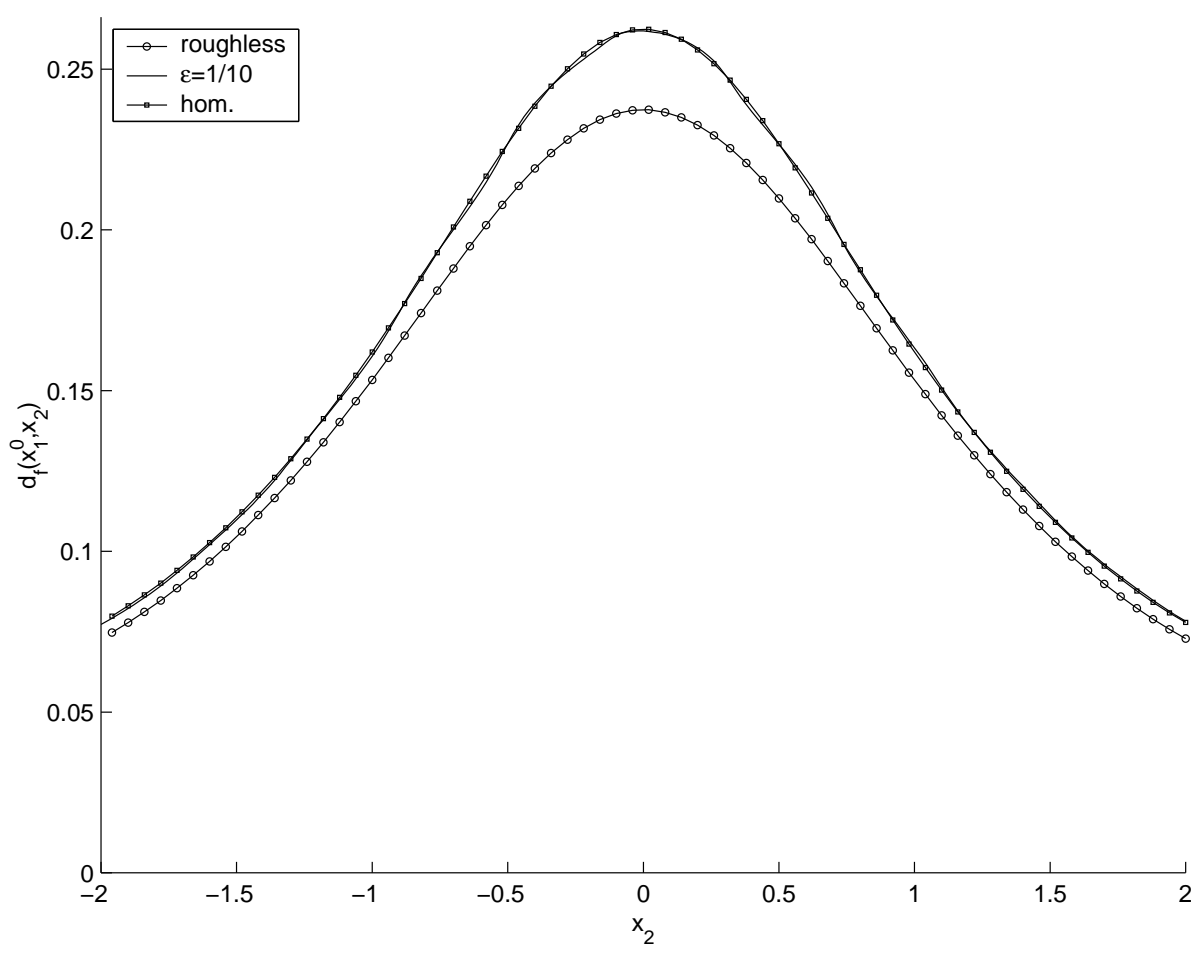

Fig. 17. EHL deformation with longitudinal roughness patterns at $x_{1}^{0}=-0.4$ 


\subsubsection{Influence of the roughness effects in EHL and hydrodynamic cases}

FIG.18 and 19 show the difference of roughness effects between a purely hydrodynamic (isoviscous) configuration and an elastohydrodynamic (piezoviscous) configuration. The data are the same as in the transverse roughness case (see Subsection 4.2.1), except for the rough gap whose amplitude of roughness patterns is modified in order to prevent contact between the surfaces in the hydrodynamic case: thus, the gap is

$$
h_{0}+\frac{x_{1}^{2}+x_{2}^{2}}{2}+0.7 h_{0} \sin \left(2 \pi \frac{x_{1}+4}{6 \varepsilon}\right)
$$

all other numerical data being the same as before (in particular for the value of $h_{0}$ and the piezoviscosity parameter $\alpha$ ). It can be noticed that the elevation of the pressure due to the roughness patterns is less important in the EHL case than in the purely hydrodynamic case. This is due to the fact that the elastic deformation tends to damp the additional load corresponding to the roughness. It has little influence over the saturation distribution, although the homogenization process does not allow to get microcavitation effects which do exist when a deterministic rough pattern is considered. Though, this analysis also states that microcavitation effects tend to vanish as $\varepsilon$ tends to 0 .

\subsubsection{Influence of the roughness over the load}

Numerical tests have been made for the following rigid contribution to the gap:

$$
h_{0}+\frac{x_{1}^{2}+x_{2}^{2}}{2}+a_{r} \sin \left(2 \pi \frac{x_{1}+4}{6 \varepsilon}\right)
$$

with $h_{0} \in\{0.5,1,1.5,2\}$ and $a_{r} / h_{0} \in\{0.2,0.4,0.6,0.8,1\}$. Moreover, the elastic contribution to the gap is the one given at the beginning of Subsection 4.2, and piezoviscosity has been taken to $\alpha=0$ (isoviscous case). Results are given on FIG.20, showing the influence of the minimum thickness $h_{r}-a_{r}$ over the load $W$ for different values of $h_{0}$. Results are taken from the analysis of the corresponding homogenized solution.

\subsubsection{Influence of the piezoviscosity}

We focus on the behaviour of the solution with respect to the piezoviscosity parameter $\alpha$. Numerical data are the same than in the elastohydrodynamic case with transverse roughness (Subsection 4.2.1), except that we take into account piezoviscous properties of the lubricant: $\alpha=0,1,2$ or 3 .

FIG.21 and 22 represent the pressure and deformation profiles at $x_{2}^{0}=0$ in the homogenized case. They illustrate the trend induced by the piezoviscosity parameter: the peak pressure and the peak deformation increase with $\alpha$. Only 


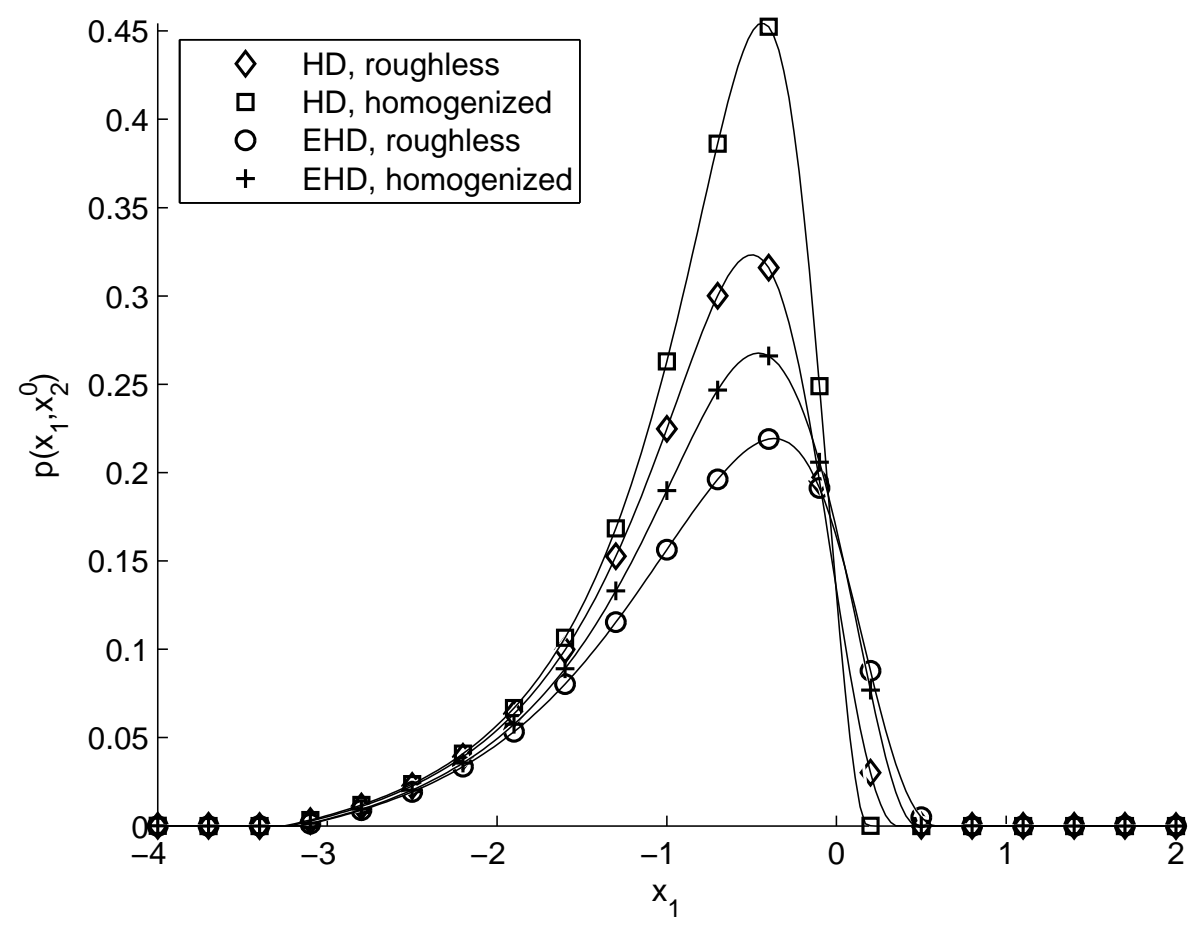

Fig. 18. Transverse roughness effects over the pressure in purely hydrodynamic and elastohydrodynamic cases at $x_{2}^{0}=0$

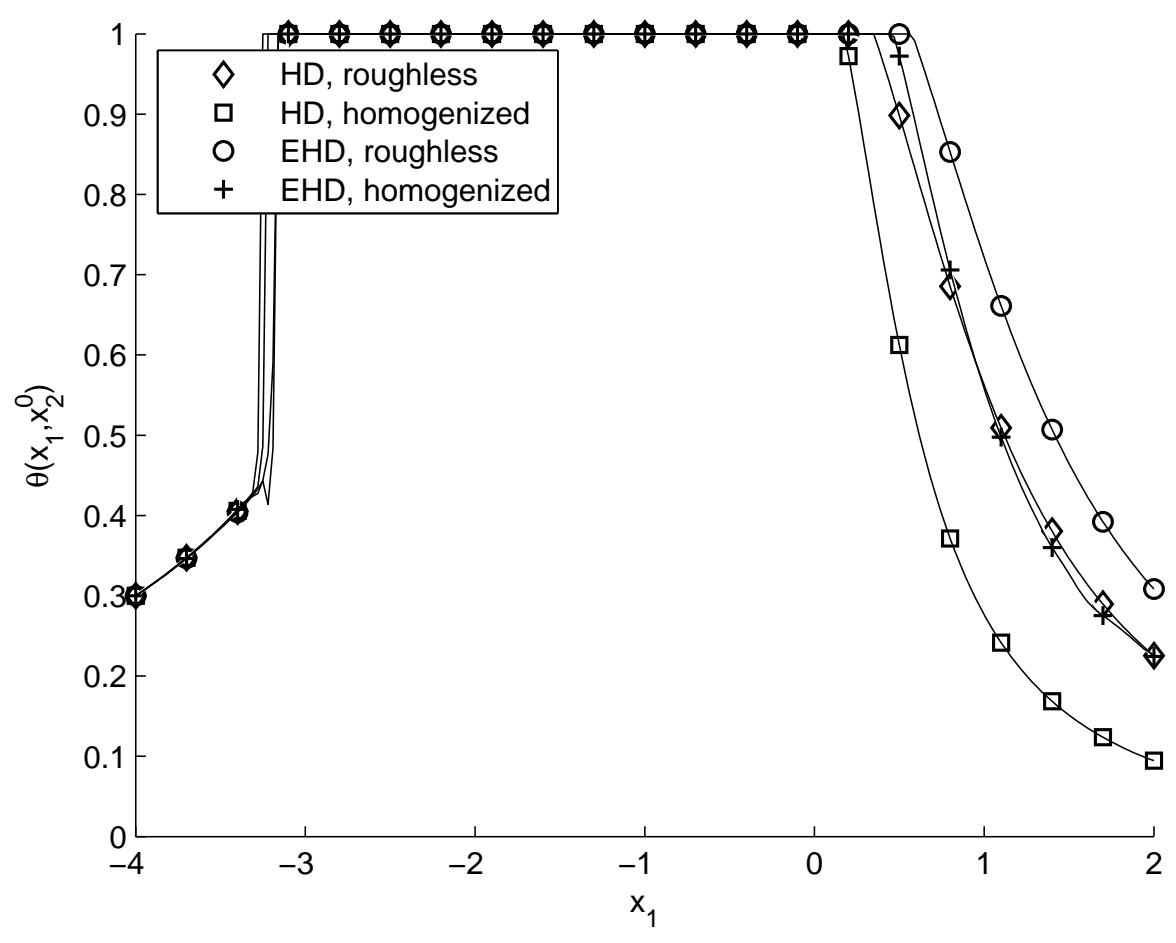

Fig. 19. Transverse roughness effects over the saturation in purely hydrodynamic and elastohydrodynamic cases at $x_{2}^{0}=0$ 


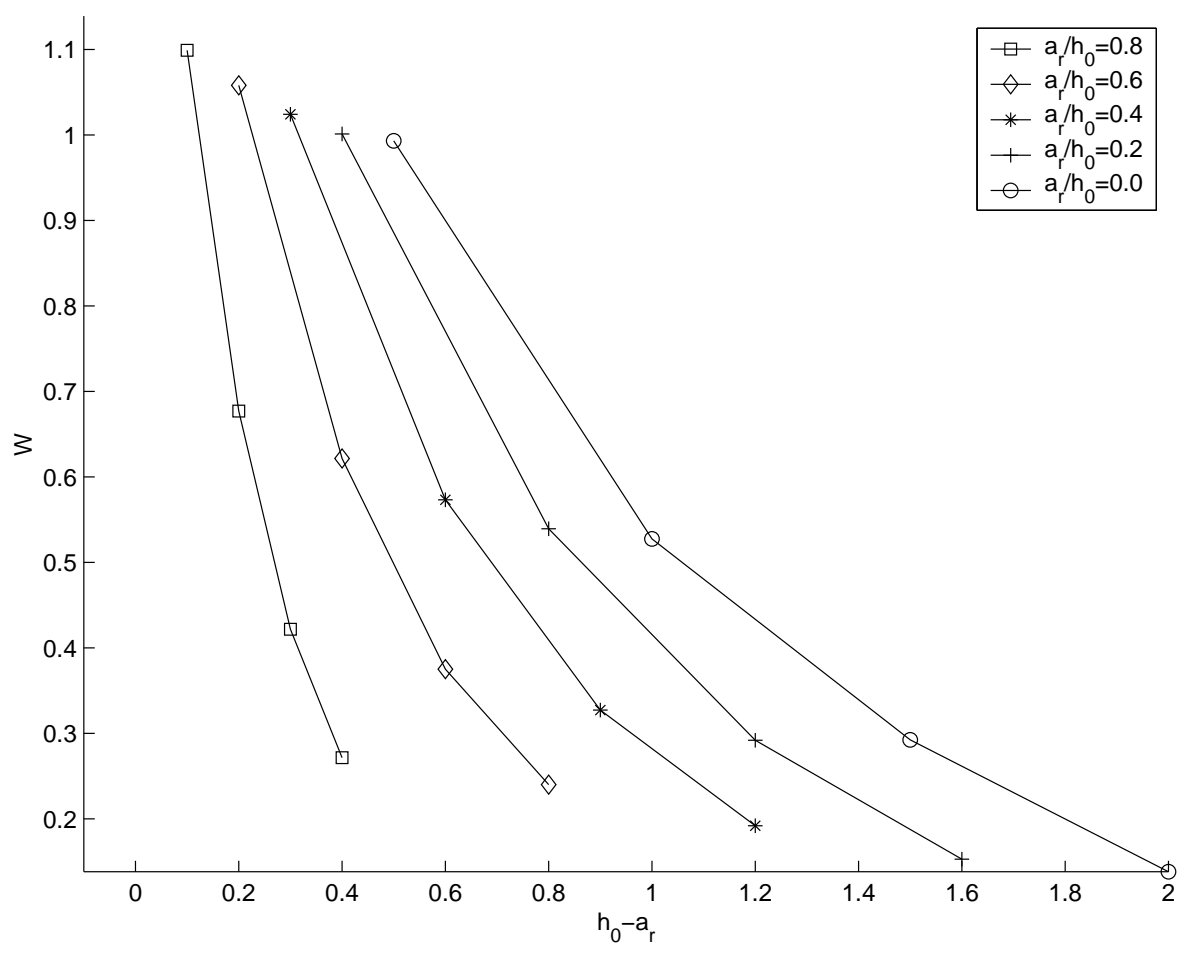

Fig. 20. Influence of the roughness over the load

a few variations affect the saturation distribution (for this reason, the corresponding curves are omitted).

TABLE 2 (resp. TABle 3) gives the variation of the peak pressure (resp. deformation) with respect to the isoviscous case $(\alpha=0)$ in different rough cases (including roughless and homogenized ones).

The relative variation of the peak pressure is denoted

$$
\Delta p / p=\frac{\max \left(p^{\alpha}\right)-\max \left(p^{0}\right)}{\max \left(p^{0}\right)}
$$

where $p^{\alpha}$ (resp. $p^{0}$ ) denotes the pressure distribution corresponding to the piezoviscous regime $\alpha \neq 0$ (resp. isoviscous regime $\alpha=0$ ). Similarly, the relative variation of the peak deformation is denoted

$$
\Delta d / d=\frac{\max \left(d_{f}^{\alpha}\right)-\max \left(d_{f}^{0}\right)}{\max \left(d_{f}^{0}\right)}
$$

where $d_{f}^{\alpha}\left(\right.$ resp. $\left.d_{f}^{0}\right)$ denotes the deformation distribution corresponding to the piezoviscous regime $\alpha \neq 0$ (resp. isoviscous regime $\alpha=0$ ). 


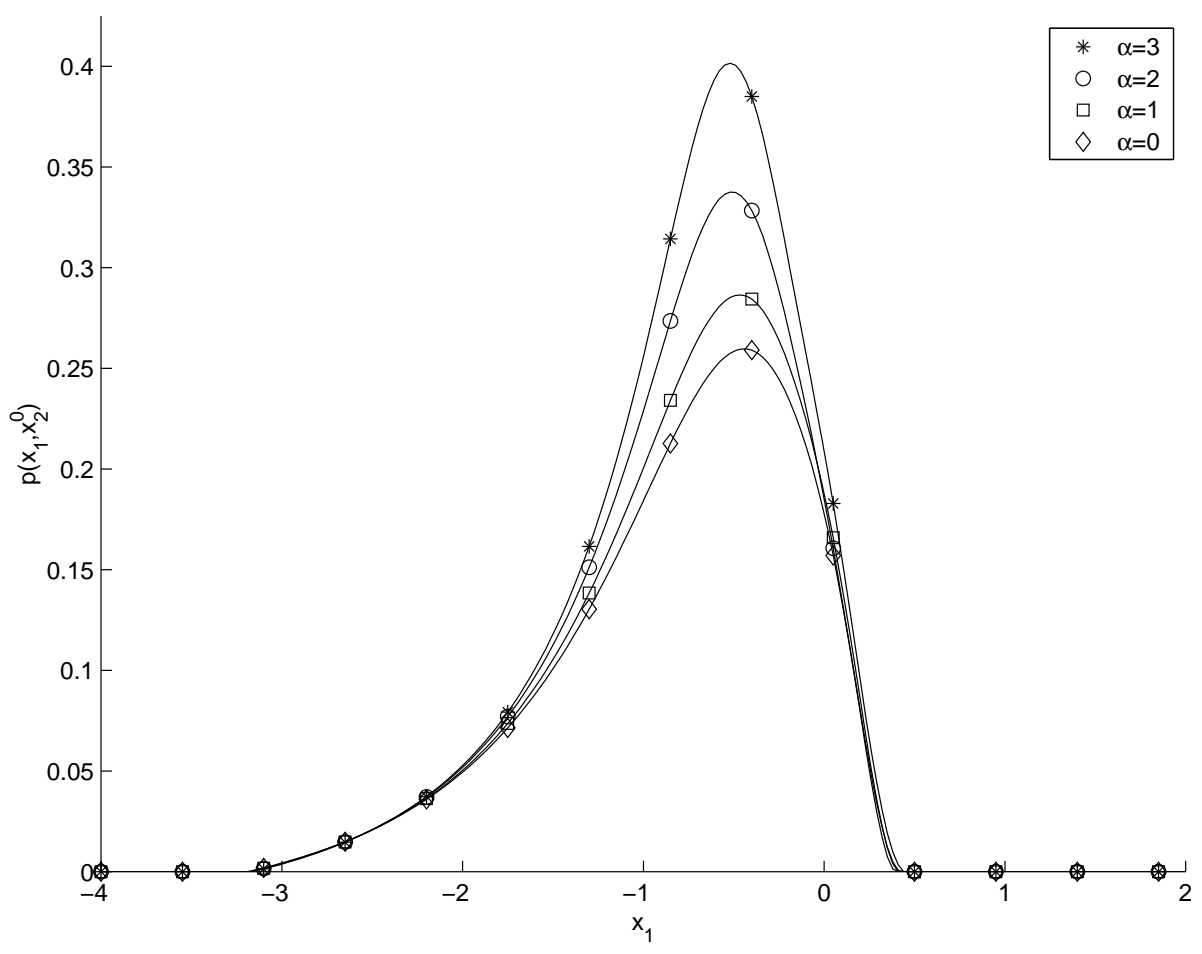

Fig. 21. Influence of the piezoviscosity over the (homogenized) pressure

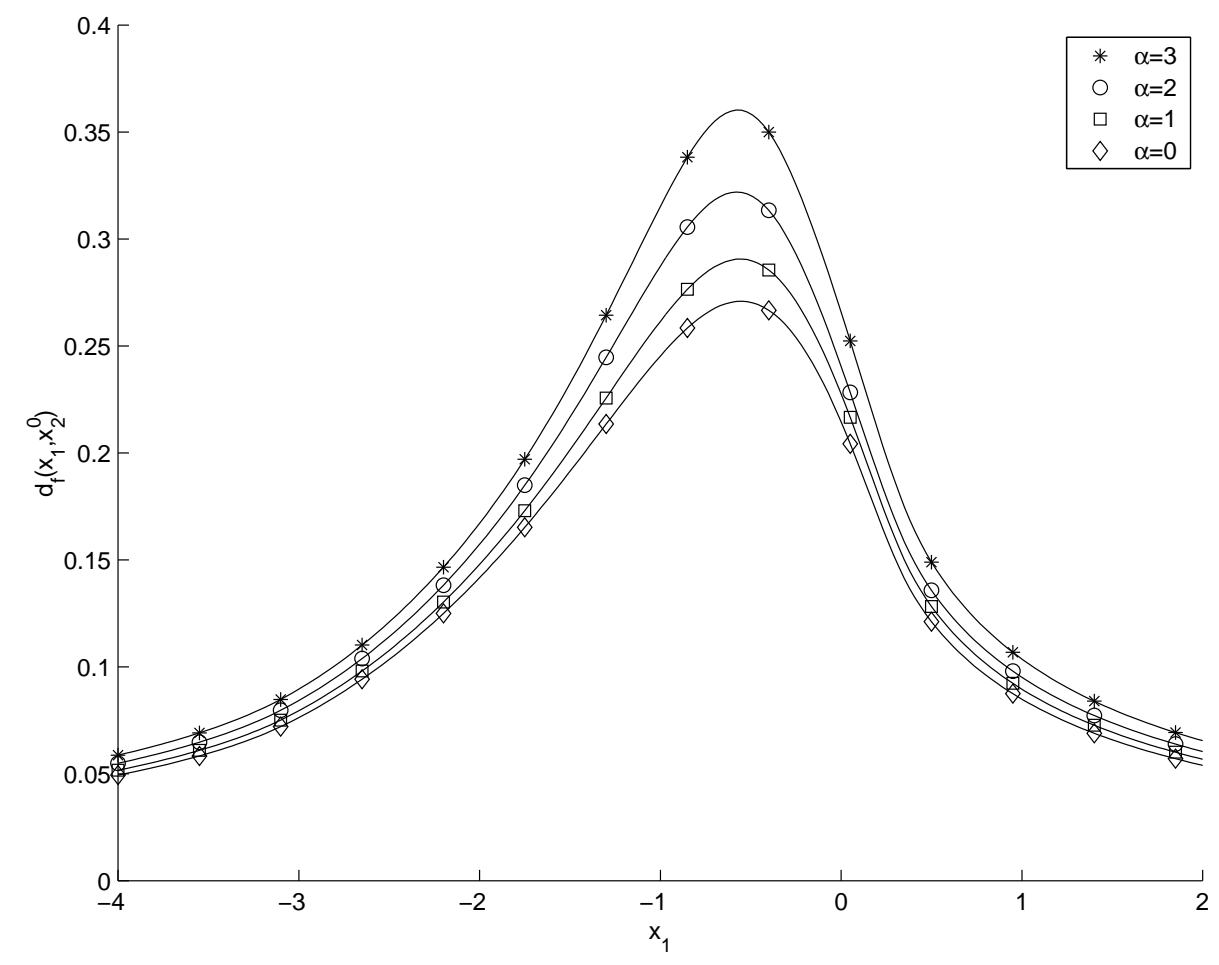

Fig. 22. Influence of the piezoviscosity over the (homogenized) deformation 
Table 2

\begin{tabular}{|c||c|c|c|}
\hline$\Delta p / p$ & $\alpha=1$ & $\alpha=2$ & $\alpha=3$ \\
\hline \hline roughless & 0.0817 & 0.2633 & 0.4449 \\
\hline$\varepsilon=1 / 10$ & 0.1035 & 1.8646 & 2.8203 \\
\hline$\varepsilon=1 / 20$ & 0.2447 & 0.6551 & 1.0192 \\
\hline$\varepsilon=1 / 30$ & 0.1855 & 0.5161 & 1.3783 \\
\hline homogenized & 0.1041 & 0.3030 & 0.5543 \\
\hline
\end{tabular}

Maximum pressure elevation due to piezoviscosity

Table 3

\begin{tabular}{|c||c|c|c|}
\hline$\Delta d / d$ & $\alpha=1$ & $\alpha=2$ & $\alpha=3$ \\
\hline \hline roughless & 0.0540 & 0.1716 & 0.2787 \\
\hline$\varepsilon=1 / 10$ & 0.0584 & 0.5742 & 0.6034 \\
\hline$\varepsilon=1 / 20$ & 0.1078 & 0.2497 & 0.4532 \\
\hline$\varepsilon=1 / 30$ & 0.0907 & 0.2198 & 0.4386 \\
\hline homogenized & 0.0732 & 0.1895 & 0.3320 \\
\hline
\end{tabular}

Maximum deformation elevation due to piezoviscosity

\section{Conclusion}

The solution procedure for deterministic periodic roughness computation, which has been developped in [3], has been extended to the (piezoviscous) elastohydrodynamic case. It is valid for transverse or longitudinal roughness patterns. Further investigation has to be made in order to take into account anisotropic two dimensional effects.

\section{References}

[1] G. Bayada, J.-B. Faure, A double-scale analysis approach of the Reynolds roughness. Comments and application to the journal bearing, ASME J. of Tribology 111 (1989) 323-330.

[2] G. Bayada, S. Martin, C. Vázquez, Effets d'anisotropie par homogénéisation dans un problème à frontière libre, C. R. Math. Acad. Sci. Paris 340 (7) (2005) $541-546$.

[3] G. Bayada, S. Martin, C. Vázquez, An average flow model of the Reynolds roughness including a mass-flow preserving cavitation model, ASME J. of Tribology (to appear). 
[4] A. Kumar, J. F. Booker, A finite element cavitation algorithm, ASME J. of Tribology 113 (2) (1991) 276-86.

[5] F. Shi, R. F. Salant, A mixed soft elastohydrodynamic lubrication model with interasperity cavitation and surface shear deformation, ASME J. of Tribology 122 (2000) 308-316.

[6] D. Vijayaraghavan, T. G. Keith, An efficient, robust, and time accurate numerical scheme applied to a cavitation algorithm, ASME J. of Tribology 112 (1990) 44-51.

[7] D. Dowson, G. R. Higginson, Elastohydrodynamic lubrication, Pergamon Press, Oxford, Great Britain, 1977.

[8] G. Bayada, M. Chambat, C. Vázquez, Characteristics method for the formulation and computation of a free boundary cavitation problem, J. Comput. Appl. Math. 98 (2) (1998) 191-212.

[9] A. Bermúdez, J. Durany, La méthode des caractéristiques pour les problèmes de convection-diffusion stationnaires, RAIRO Modél. Math. Anal. Numér. 21 (1) (1987) 7-26.

[10] I. Arregui, J. J. Cendán, C. Vázquez, Mathematical analysis and numerical simulation of a Reynolds-Koiter model for the elastohydrodynamic journalbearing device, M2AN Math. Model. Numer. Anal. 36 (2) (2002) 325-343.

[11] J. Durany, G. García, C. Vázquez, Numerical simulation of a lubricated Hertzian contact problem under imposed load, Finite Elem. Anal. Des. 38 (7) (2002) 645-658. 